\title{
Detection efficiency and photometry in supernova surveys
}

\section{The Stockholm VIMOS Supernova Survey I}

\author{
J. Melinder ${ }^{1}$, S. Mattila ${ }^{2,3}$, G. Östlin ${ }^{1}$, L. Mencía Trinchant ${ }^{1}$, and C. Fransson ${ }^{1}$ \\ 1 Stockholm Observatory, AlbaNova University Centre, 10691 Stockholm, Sweden \\ e-mail: jens@astro.su.se \\ 2 Tuorla Observatory, University of Turku, Väisäläntie 20, 21500 Piikkiö, Finland \\ 3 Astrophysics Research Centre, School of Mathematics and Physics, Queen's University Belfast, Belfast BT7 1NN, UK \\ Received 6 December 2007 / Accepted 30 June 2008
}

\section{ABSTRACT}

\begin{abstract}
Aims. The aim of the work presented in this paper is to test and optimise supernova detection methods based on the optimal image subtraction technique. The main focus is on applying the detection methods to wide field supernova imaging surveys and in particular to the Stockholm VIMOS Supernova Survey (SVISS).

Methods. We have constructed a supernova detection pipeline for imaging surveys. The core of the pipeline is image subtraction using the ISIS 2.2 package. Using real data from the SVISS we simulate supernovae in the images, both inside and outside galaxies. The detection pipeline is then run on the simulated frames and the effects of image quality and subtraction parameters on the detection efficiency and photometric accuracy are studied.

Results. The pipeline allows efficient detection of faint supernovae in the deep imaging data. It also allows controlling and correcting for possible systematic effects in the SN detection and photometry. We find such a systematic effect in the form of a small systematic flux offset remaining at the positions of galaxies in the subtracted frames. This offset will not only affect the photometric accuracy of the survey, but also the detection efficiencies.

Conclusions. Our study has shown that ISIS 2.2 works well for the SVISS data. We have found that the detection efficiency and photometric accuracy of the survey are affected by the stamp selection for the image subtraction and by host galaxy brightness. With our tools the subtraction results can be further optimised, any systematic effects can be controlled and photometric errors estimated, which is very important for the SVISS, as well as for future SN searches based on large imaging surveys such as Pan-STARRS and LSST.
\end{abstract}

Key words. supernovae: general - methods: data analysis - surveys

\section{Introduction}

Imaging surveys to find and characterise variable sources are used to study a number of different astronomical phenomena. Detection and photometry of variable sources can be done by subtracting images taken at different epochs from each other and analysing the subtracted frames, a technique known as difference imaging. In addition to supernovae ( $\mathrm{SNe}$ ), this technique has been used by various authors investigating many different subjects, among which are variable stars in crowded fields (e.g. Corwin et al. 2006), gravitational micro-lensing (e.g. Alard 2001; Sumi et al. 2006) and planet detection (e.g. Holman et al. 2007).

The most complicated step in the difference imaging process is taking into account the spatial and temporal variability of the point spread functions (PSFs) of the images. The difference imaging technique used in this work is based on the Optimal Image Subtraction (OIS) code first presented in Alard \& Lupton (1998, hereafter AL98), later improved upon in Alard (2000, hereafter A00) and available in the ISIS 2.2 package. In these papers the code is tested on variable stars (i.e. point sources with varying brightness with little or no background light). When detecting $\mathrm{SNe}$ the situation can be quite different. In this type of

* Based on observations collected at the European Organisation for Astronomical Research in the Southern Hemisphere, Chile, under ESO programme ID's 167.D-0492(C) and 167.D-0492(D). data the background light from the host galaxy often dominates the total flux at the location of the supernova.

Difference imaging, and OIS in particular, has been used to detect $\mathrm{SNe}$ in many projects. Recently a number of large SN surveys, mainly aiming at finding thermonuclear $\mathrm{SNe}$ for determining cosmological parameters, have released their first results. Image subtraction using the OIS algorithm followed by detection of SN candidates in the subtracted frames is a technique that has been used by the ESSENCE survey (the observations and data analysis are described in Miknaitis et al. 2007), the Supernova Legacy Survey (Astier et al. 2006) and Poznanski et al. (2007). Cappellaro et al. (2005) and Botticella et al. (2008) used the same technique in their SN surveys aiming to determine the core collapse SN rate at intermediate redshift. Difference imaging has also successfully been used in near-infrared SN searches (e.g. Mattila \& Meikle 2001; Maiolino et al. 2002) concentrating on individual starburst galaxies. For surveys with the aim of finding supernova rates the detection efficiency is the most important issue, while the SN photometry is of somewhat less importance. However, for surveys targeting Ia SNe the photometry is of utmost importance since the photometric accuracy directly determines how well the cosmological parameters can be constrained. Therefore, in these programmes the photometry is treated very carefully and not necessarily obtained from the subtracted frames. For studies of individual SNe difference imaging has been used to obtain accurate photometry of supernovae with 
a complicated background. This is particularly important at late times when the SN has become too faint compared to the background host galaxy in the unsubtracted image for PSF photometry to give reliable results (e.g. Sollerman et al. 2002). More recently, difference imaging has also successfully been applied to mid-infrared SN observations with the Spitzer Space Telescope (Meikle et al. 2006) and to adaptive optics assisted detection of SNe in luminous infrared galaxies (Mattila et al. 2007).

In this paper we investigate several aspects of difference imaging with ISIS 2.2 when used to detect and study supernovae. Previous papers have already reported some tests done on the image subtraction (e.g., AL98; A00; Israel et al. 2007) and on the SN detection process (e.g. Mattila \& Meikle 2001). The surveys for thermonuclear SNe all have extensive sections where the methods to ascertain the photometric accuracy (both in terms of statistical and systematic errors) is described (e.g. Astier et al. 2006). However, no systematic tests on the subtraction method applied to supernova data have been reported. The main purpose of this paper is to describe our supernova detection pipeline and to study how it performs on wide field imaging surveys, in particular the Stockholm VIMOS Supernova Survey (SVISS). The SVISS is an imaging survey aiming to find corecollapse supernovae at $0.1<z<1.0$ (for details see Dahlén \& Fransson 1999). The main scientific goal for this project is to find reliable core collapse supernova rate densities for this redshift range. We thus need to have detailed knowledge of the supernova detection efficiency. In this project the photometric accuracy is important to correctly type the SNe based on colour and light curve information. Another goal of this paper is to investigate how supernova detection and photometry (with ISIS used as the image subtraction tool) are affected by the selection of subtraction and detection parameters and the presence of a host galaxy. In this paper we discuss the subtraction and detection method, in the following papers we will present the light curves of the detected $\mathrm{SNe}$, the typing methodology and calculations of $\mathrm{SN}$ rates at high redshifts.

The first part of the paper (Sect. 2) contains a detailed description of the standard image subtraction method used in this work. In Sect. 3 we describe the data sets, in Sect. 4 how the simulations of supernovae have been setup and in Sect. 5 we present the results from the extensive testing. Finally, we discuss the results and possible implications in Sect. 6 and conclude by giving a short summary in Sect. 7. The Vega magnitude system has been used throughout the paper.

\section{Image subtraction and SN detection method}

We have constructed a supernova detection pipeline suitable for large images and multi-epoch data. The pipeline consists of a number of IRAF/PYRAF scripts that are run in sequence, and go through the following steps: (i) accurate image alignment over the entire frame; (ii) convolving the better seeing image to the same PSF size and shape as the poorer seeing image, using a spatially varying kernel; (iii) subtracting the images; (iv) detection of sources in the subtracted frames, using both source detection software and eye-ball detection; (v) photometry and construction of light curves of the detected objects.

The input to the pipeline consists of one reference image and a list of later epoch images. The reference image is subtracted from each of the later epoch images and objects with varying brightness are detected in the first search epoch image. The output from the pipeline is a list of supernova candidates detected in the first search epoch and a light curve constructed from measurements on the detected position in the subsequent epoch images. The detection part of the pipeline can then be rerun with the previous second epoch as detection epoch (enabling SNe that have appeared between the first two epochs to be found). This can be repeated for all search epochs, i.e. once a particular search epoch has been run through the pipeline, that epoch can then be used as a reference epoch for subsequent epochs. This is useful when a search epoch image is of better quality (in terms of seeing and depth) than the reference epoch image.

\subsection{Registering images}

The subtraction method is quite sensitive to how well the images are registered. Spatial variability of the convolution kernel can somewhat compensate for a non-perfect image alignment (also discussed in Israel et al. 2007), but in general the images should be aligned with high precision before running the actual subtraction. Accurate image alignment is particularly important when the seeing differences are small, since narrow convolution kernels will not be able to compensate for poor image alignment.

All the input images are aligned to the reference image by using the IRAF tasks geomap and geotran. A number of bright sources visible in all of the frames are used as input coordinate lists for these tasks. The exact positions of the sources are found by using the IRAF task imcentroid. The number of reference sources used is dependent on the order of the geometrical transformation and the size of the image.

\subsection{PSF matching and image subtraction}

The atmospheric conditions will affect the width and shape of the PSFs of the images. The combination of the atmospheric effects with the optics, focusing and camera setup results in variations of the PSF of the images both in spatial coordinates and over time (i.e. different epochs can have different PSFs, and also different spatial variation). A successful image subtraction technique must be able to match the PSF of the reference image to the PSF of the later epoch images also allowing the convolution kernel to vary spatially. The OIS technique presented in A00 allows this.

Following A00 (using the same notation) we summaries the OIS technique. The principal difficulty with image subtraction is to find a convolution kernel $(K)$ that can transform the PSF of a reference image $(R)$ to the PSF of an arbitrary image $(I)$. The best-fit kernel can be found by minimising the sum over all pixels

$$
\sum_{i}\left([R \otimes K]\left(x_{i}, y_{i}\right)-I\left(x_{i}, y_{i}\right)+b g\left(x_{i}, y_{i}\right)\right)^{2},
$$

where $b g\left(x_{i}, y_{i}\right)$ is a spatially varying background term. The kernel can be written as a sum of basis functions

$K(u, v)=\sum_{n} a_{n}(x, y) K_{n}(u, v)$

where $a_{n}$ contain the spatial variations of the kernel, which are polynomial functions of a given degree. $K_{n}$ are the basis functions for the kernel and $u$ and $v$ denote the PSF kernel coordinates. The basis functions are Gaussian functions of the type

$K_{n}(u, v)=\mathrm{e}^{-\left(u^{2}+v^{2}\right) / 2 \sigma_{k}^{2}} u^{i} v^{j}$

with the generalised index $n=(i, j, k)$. The basis functions can have different widths (the $k$ index) and different orders (the $i$ and $j$ indices). To deal with a spatially varying background a polynomial background term is present in Eq. (1) and is also included in the solution which is found by solving the resulting linear system 
Table 1. Default subtraction parameters.

\begin{tabular}{lc}
\hline \hline Parameter & SVISS data set value \\
\hline Subregions & 9 \\
Total number of stamps $\left(n_{\text {st }}\right)$ & $\sim 450$ \\
Size of the kernel $(\times F W H M)$ & 4.0 \\
Full size of the stamp $(\times F W H M)$ & 6.0 \\
Fitting order for the background & 2 \\
Degree of kernel spatial variation & 1 \\
Orders of the Gaussian basis functions & $6 / 4 / 3$ \\
\hline
\end{tabular}

(for details see A98 and A00). The reference image is convolved with the kernel and subtracted from the search image.

The default parameters for image subtraction used for the SVISS images are listed in Table 1. The basis functions described above used in ISIS 2.2 are three Gaussian functions of orders 6, 4 and 3. Both Israel et al. (2007) and Gössl \& Riffeser (2002) find that the use of 3 Gaussian functions of these orders works well, we use these basis functions in our subtractions. We have, however, done some tests with different widths of the functions which is reported in Sect. 5.3. The order of the polynomial functions used for the spatial variation of the kernel in A00 is 2. Gössl \& Riffeser (2002) use a zero order spatial variability, but compute local kernels for subregions in the image. For the SVISS data set we subdivide the large $2 \mathrm{k} \times 2.5 \mathrm{k}$ pixels VIMOS frames into 9 subregions and use a first order spatial variation of the kernel. We have chosen to use kernel and stamp sizes scaled for the poorer seeing image (i.e. the search image), however we have not found these sizes to affect the subtraction quality substantially. A very small kernel size (smaller than the $F W H M$ of the image) will likely cause the subtraction to fail. A too large kernel size will on the other hand include more noise in the fit and probably make the subtraction quality worse. In general, varying the parameters listed in Table 1, have considerably smaller effect on the resulting subtraction quality than the selection of stamps, data quality and host galaxy brightness (see Sect. 4.3).

In ISIS a number of sources present in both reference and search image are used as the data that goes into the kernel solving procedure. A number of high $\mathrm{S} / \mathrm{N}$-sources are selected from the image and a small subimage (so called stamp) is obtained for each of them. The selected sources do not have to be point sources, but they have to be non-saturated and well below the non-linear response regime of the detector. The stamps are selected by dividing the image into a number of rectangular sections (as many as the wanted number of stamps). In each of these sections the brightest non-saturated pixel is found and the stamp is centred on that pixel. To check that no variable objects are selected, the fluxes inside the stamps in the two images are compared and the variable stamps are rejected.

Our initial test runs showed some problems with the automatic stamp selection. The stamp selection is very sensitive to how saturated/bad pixels are handled in the data, if not treated correctly saturated objects could be selected as stamps. When simulating bright $\mathrm{SNe}$ we find that the simulated sources themselves are often selected as stamps even though they are only present in one of the frames, and cause the subtraction quality to deteriorate. This indicates that the rejection routine described above is not working for our data set with simulated $\mathrm{SNe}$. However, often the number of SNe per image is expected to be quite low, thus the effect on real survey data is expected to be marginal. In our simulations, where a large number of artificial $\mathrm{SNe}$ were placed in the images the effect is clearly noticeable.
To gain a better control over the stamp selection process we have modified ISIS to accept an external stamp list. This version of ISIS has previously been used for SN detection in Mattila \& Meikle (2001) and Mattila et al. (2004, 2007) with manual stamp selection. In this study the stamps are selected by running SExtractor 2.4.4 (Bertin \& Arnouts 1996) on the reference image, which allows us to reject stamps that are too faint and to place constraints on the stellarity of the sources. In this way we can also reject stamps that are suspected to contain a variable source and then rerun the subtractions. The stamp region list can also be compared with the bad pixel masks for both the reference and the detection image to make sure that no stamp regions contain saturated pixels.

In supernova surveys the sources selected as stamps are not necessarily point sources because the number of available bright point sources is simply too small. The majority of the automatically selected stamps will be extended sources, so if point sources are preferred the external stamp list method has to be used. In Sect. 5.4 we have examined the effects of different selection criteria for the stamps and how the number of stamps affects the subtractions.

\subsection{Source detection}

Source detection is done in the subtracted frames using SExtractor (SE) to get an initial source list. The frames are also manually inspected to check for possible image defects and obvious spurious detections (e.g., sources close to saturated objects). The detection thresholds in SE are set by giving the minimum area of detected objects in pixels (MIN_AREA) and the minimum flux for a pixel to be considered detected (DET_THRESH). We have used MIN_AREA parameter corresponding to a circular area with diameter of roughly $1 \times F W H M$. The DET_THRES parameter is a multiplier to the measured pixel to pixel noise (rms) in the frame, this parameter has been adjusted to detect faint sources while still keeping the number of spurious detections down (see Sect. 4.4.4 for a discussion on this). Convolving the images affects the noise and therefore also the optimal detection threshold to use, and since we are using other methods to ensure that spurious detections are excluded we have chosen to use a low detection threshold $(1.5 \times$ the $\mathrm{rms}$ measured in the subtracted frame).

In SE weight-maps in the form of variance maps can be used to correct the detections for varying noise levels over the image (due to e.g. vignetting and exposure time differences). This is especially helpful in the edges of images. Using the SE option of WEIGHT_TYPE set to MAP_WEIGHT our exposure time maps are scaled to inverted variance maps within SE. The inclusion of a weight-map in the detection decreases the amount of spurious detections close to the edges of the subtracted images considerably. For each epoch weight-maps in the form of exposure time maps can be constructed (see 3 for a description on how this was done for the SVISS data set). For counts-persecond CCD images where the instrumental noise is negligible (i.e. the noise is dominated by photon noise from the source or sky) the standard deviation of the individual pixel flux scales as the square root of the exposure time. The pixel noise in the subtracted image is approximately given by quadrature addition of the reference and search image noise (AL98). Convolving the reference image slightly changes the noise characteristics of the subtracted image (discussed in A00), but for constructing the combined weight-maps we have found it sufficient to use 
the unconvolved reference exposure time maps. The combined weight-maps are then computed as follows:

$W_{\mathrm{c}}=\frac{1}{\frac{1}{W_{\text {ref }}}+\frac{1}{W_{\mathrm{s}}}}$,

where $W_{\text {ref }}$ and $W_{\mathrm{s}}$ are the exposure time maps (weight-maps) for the reference image and the search image respectively and $W_{\mathrm{c}}$ is the weight-map for the subtracted image. Note that the weight maps are only used in the detection step in SE, photometry is done on the unweighted frame.

All of the detections with valid photometry (in this case errors lower than $1.0 \mathrm{mag}$ ) are kept. The coordinates of the detected sources are all put into a master candidate list, this is then used for the photometry (described in the next section). The master candidate list can also be used to do multi-epoch and multiband detection of the sources found in the search epoch image under consideration. The master candidate list will contain a large number of spurious detections (due to the low detection threshold used and the presence of subtraction residuals). These objects can be excluded by combining three different criteria; (i) too faint, the sources are fainter than a predefined limit (e.g. signal to noise limit from simulations); (ii) only present in one filter; (iii) light curve not consistent with SN templates.

\subsection{Photometry of variable sources}

Photometry on the detected sources was done using the IRAF daophot package. PSF photometry was done on all the detected candidates using the task allstar. The PSF model used for each subtracted frame was constructed from the original worse seeing image (the search image) using the task psf. In this task point sources are fitted with model profiles and the profile yielding the smallest residuals is chosen as the best PSF fit. The order of variability of the PSF model was set to zero, i.e. the PSF was not allowed to vary over the frame. Note that the model was constructed from the real data, thus the shape of the PSF is not symmetrical and does contain noise. As discussed earlier, often the PSF vary over the frames. However, using a higher order variability in the psf photometry did not result in any gain in the photometric accuracy. We also used aperture photometry with aperture corrections computed from the original worse seeing image, using the IRAF task phot. This does give fairly good results, but seems to be more susceptible to residual flux from the background galaxies than the PSF photometry, thus giving larger errors (both statistical and systematic) in general.

Using the pixel to pixel noise in the subtracted image will not give a correct estimate of the photometric errors, the pixels will be positively correlated due to the convolution of the reference image. Another problem in using the pixel to pixel noise estimate is that it will in general be computed in an annulus outside the aperture (or outside the size of the PSF model in the case of PSF photometry). The annulus region for most $\mathrm{SNe}$ will be outside the host galaxy, at least in the SVISS case where most host galaxies are just barely resolved. The noise estimate in this region can in principle be quite different from the noise at the SN position where residuals from the subtracted host galaxy will also be present. Both these effects result in the noise estimates given by daophot and phot underestimating the true photometric errors severely. Instead we use the simulations described in Sect. 4 to obtain reliable error estimates. By simulating $\mathrm{SNe}$ at different brightness in each epoch and finding the scatter in their measured magnitudes, we obtain an estimate of the true photometric error for each point on the SN light curve.
Table 2. Data sample overview.

\begin{tabular}{lcccl}
\hline \hline Data set/Epoch & Exp. time $(\mathrm{s})$ & $m_{\text {lim,rms }}{ }^{\dagger}$ & $m_{\text {lim,sim }} \ddagger$ & Seeing $\left({ }^{\prime \prime}\right)$ \\
\hline SVISS/Ref & 11520 & 26.36 & 26.1 & 0.61 \\
SVISS/A & 11520 & 26.64 & 26.3 & 0.68 \\
SVISS/B & 9600 & 26.48 & 26.1 & 0.72 \\
SVISS/C & 14400 & 26.62 & 26.3 & 0.64 \\
SVISS/D & 7680 & 26.61 & 26.2 & 0.65 \\
\hline
\end{tabular}

$\dagger$ The limiting magnitudes are $3 \sigma$ limits and have been measured for an aperture of diameter $4 \times$ the $F W H M$.

$\$$ The limiting magnitudes are $3 \sigma$ limits and have been estimated from the photometric scatter of simulated sources in the individual unsubtracted epoch images.

The limiting magnitude for a given epoch depends on the depths of both the reference and the search images. Since the noise in the subtracted image will be approximately equal to quadrature added noise of the images, the subtracted image will in general have a higher level of noise by a factor $\sqrt{2}$ and therefore be $\sim 0.4$ mag less deep than the individual images. For the SVISS data, this is true for the pixel-to-pixel noise. The subtracted frames do have on the order of $\sqrt{2}$ higher pixel rms (but slightly lower due to the convolution). However, we have found that the limiting magnitudes computed for the individual SVISS epochs based on pixel-to-pixel noise ( $m_{\text {sim,rms }}$ in Table 2$)$ are not representative of the true depth as measured by by our simulation technique. We have measured the photometric scatter of the simulated $\mathrm{SNe}$ in the unsubtracted epoch images and obtained more conservative depth estimates ( $m_{\text {sim,sim }}$ in Table 2$)$.

The reason why the photometric scatter estimate results in larger errors than the simple pixel-to-pixel estimate is a combination of several factors. We believe that the main factor is that our deep images are coming close to the confusion limit. In photometry the subtraction of a local background becomes very complicated when the background is allowed to vary over sizes comparable to the aperture size (or fitting radius for the PSF magnitudes). The background sources just at the confusion limit inside the photometric aperture/fitting region and background annulus will vary from one simulated source to the another and can thus cause the scatter from the measured magnitudes to go up. Incorrect background subtraction in the presence of large scale background variations also seem to be a contributing factor, although of lesser importance than the one described above. It is worth noting that subtracting the images actually serves to remove these two factors making the photometric scatter go down. In reality this effect is countered by the increase in pixel-to-pixel noise caused by the subtraction, see the discussion in Sect. 5.1.

\section{The SVISS data sample}

In this section we describe the data set that was used to investigate the efficiency of the subtraction method. The data were obtained with the VIMOS instrument (LeFevre et al. 2003) mounted on the ESO Very Large Telescope (UT3) at several epochs. The reference epoch was obtained in autumn 2003 and the search epochs autumn 2004. The VIMOS instrument has four CCDs, each $2 \mathrm{k} \times 2.4 \mathrm{k}$ pixels with a pixel scale of $0.205^{\prime \prime} / \mathrm{pxl}$, covering a total area of $4 \times 56$ sq. arcmin. The SVISS observations were obtained in two fields, covering parts of the Chandra Deep Field-South (Giacconi et al. 2001) and the ELAIS-S1 field (La Franca et al. 2004), and in 5 broad band filters ( $U, B, V, R$ and $I$ ). The supernova search filters are $R$ and $I$, with roughly 
twice the exposure time in $I$ compared to $R$. Observations in these filters have been divided into 1 reference epoch (henceforth epoch 0 ) and 7 search epochs. The time difference between epoch 0 and epoch 1 is about one year and the search epochs are separated by a month.

The data used in this work is a subsample of the SVISS, the simulations have been carried out in the deepest search band (the $I$ band) and only on one of the four CCDs. The $R$ band has only been used to determine the spurious detection ratio (see Sect. 4.4.4). Table 2 contains an overview of the $I$ band data used in the tests. It should be noted that the total number of frames in the reference epoch is $\sim 80$, we have only used the best frames to construct the reference frame in order to optimise the resulting seeing. The large number of frames available for making a reference image is not by design, most of the frames were obtained in a previous failed observation period (the VIMOS instrument was taken off-line due to technical problems in fall 2003). In this paper we do not discuss situations where the reference epoch has worse seeing than the search epochs. In the full SVISS data set we have some epochs with better seeing than the reference epoch. However, we have not used those epochs in the tests reported in this paper. More details on the data and data reduction, along with source catalogues for the fields will be given in a forthcoming paper (Mencia-Trinchant et al., in prep).

The data were reduced using a data reduction pipeline for SVISS developed by our team. The data were bias subtracted, flatfielded and scaled to correct for extinction. The images were also normalised to counts per second. A bad pixel mask was produced for each of the images, containing vignetted $(<5 \%$ in most cases), saturated and cosmic ray flagged pixels.

The VIMOS I-band suffers from rather severe effects of fringing, and care has to be taken to successfully remove these. We constructed a fringe map for each observation night by median combining the non-aligned science frames and rejecting bright pixels by a standard sigma-rejection routine. The fringe map was then subtracted from each of the flatfielded science frames. All of the science frames were also manually inspected, some frames were removed because of vignetting and/or poor seeing (see Table 2 for the total number of frames used in each epoch).

The good quality frames for each epoch were registered to a common pixel coordinate system using a simple shift and rotate transform (yielding a typical $\mathrm{rms}$ of $\sim 0.1 \mathrm{pxls} / 0.02^{\prime \prime}$ ). Finally, the registered frames were median combined. The bad pixel maps for each individual image were also registered and summed, yielding an exposure time map for each epoch. Some pixels $(<0.2 \%$ in the reference epoch) had an exposure time of 0 , i.e. none of the individual frames contained useful data for that pixel. These pixels were flagged in the combined science frames.

Each of the combined epoch images were photometrically calibrated using secondary standard stars in the field. The calibrated photometry for these stars were obtained from stacked one-night images from nights where standard star observations were available.

For the reference image, a host galaxy catalogue was constructed using SE. A total of 2660 galaxies were detected, rejecting all sources with photometric errors larger than 0.1 mag and sources close to masked regions. For each of the galaxies a flux profile was calculated for four radii containing respectively $20 \%$, $40 \%, 60 \%$ and $80 \%$ (hereafter $r_{20}, r_{40}, r_{60}$ and $r_{80}$ ) of the total flux as measured by SE's AUTO photometry.

The search epochs were aligned to the reference epoch following the procedure outlined in Sect. 2.1. To obtain the necessary accuracy for the registration we needed to use a transformation that allowed separate shifts, rotations and scales for the $x$ and $y$ coordinates. The reference sources for registration were bright, point-like objects in the field (approximately 100 sources were used). The resulting rms of the geometrical transformations were smaller than 0.1 pixels for all the epochs.

The subtraction pipeline has a number of input parameters, which need to be adapted to the data set. Table 1 contain the list of parameters and the values for the SVISS data set. Since the VIMOS images are quite large we found it preferable to divide the images into subregions before the actual subtraction, which makes it possible to use a low degree of spatial variation in the kernel. The total number of stamps given in Table 1 is for the entire image. If the number of stamps was too small the subtraction quality degraded considerably. Since the number of stamps need to be tuned to the particular data set, we do not report any tests on varying this parameter. The parameters related to the size of the stamp regions have not been found to affect the subtraction quality significantly. However, no sub-FWHM sizes have been considered. The parameters given in Table 1 have been kept constant in all the reported tests.

Figure 1 show two supernova candidates detected in the $I$ band using the subtraction parameters discussed above. The bright SN candidate (upper panel in the figure) has an approximate $I$ band magnitude of 22.7 at peak and is situated inside a bright, tentatively elliptical, galaxy. The faint candidate (lower panel) has an approximate $I$ band magnitude of 24.8 at detection, and the explosion date for this candidate is not very well constrained. The host galaxy for this $\mathrm{SN}$ is fainter and more representative of average galaxies in our images. These detections demonstrate that the use of our subtraction pipeline enables us to find faint supernovae against the bright background emission from the host galaxies.

\section{Simulations}

\subsection{The supernova point spread function model}

The construction of the artificial PSF model and the addition of SNe to image frames has been done with the IRAF package daophot (see also Sect. 2.4).

For the SVISS data set approximately 40 bright star-like objects were selected by visual inspection. About half of these objects were rejected in the fitting procedure. The total number of point sources used for constructing the PSF model for each image was thus approximately 20 . The best fitting profile was found to be an elliptical Moffat profile with $\beta=2.5$. The $F W H M$ of the models for different epochs/data sets can be found in Table 2.

Supernovae of different brightness were simulated in the search images. In all test runs the $\mathrm{SNe}$ were added using the task addstar. The input model for this task was the best-fitting PSF model and the stars were added in the search image, i.e. the image with worse seeing. A total of 17 mag bins were used, the brightest bin roughly corresponds to the $100 \sigma$ limiting magnitude and the faintest bin somewhat fainter than the unsubtracted image limiting magnitude (see Table 2). The magnitude bins used are: $22.0,22.5,23.0,23.5,24.0,24.25,24.5,24.75,25.0$, $25.25,25.5,25.75,26.0,26.25,26.5,26.75$ and 27.0 .

\subsection{Placing the artificial $\mathrm{SNe}$}

When adding artificial supernovae to the images care must be taken that the subtraction process itself is not affected. This puts some constraints on how many SNe can be placed in each image, and also where they are placed. Since ISIS relies on the 


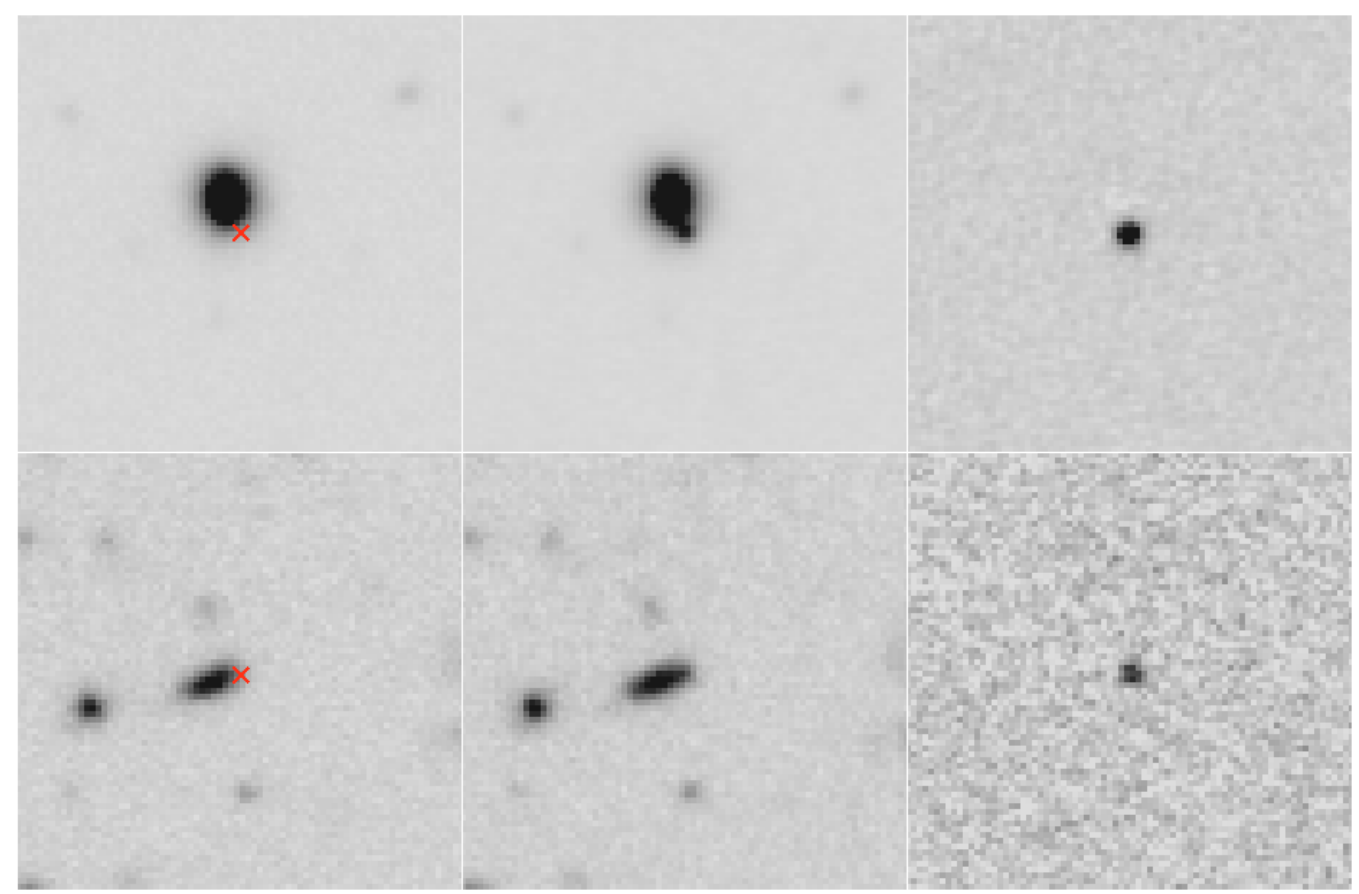

Fig. 1. Two example supernova candidates detected in the SVISS. Upper and lower panels show the two candidates with the leftmost panels showing the host galaxy in the reference image, the middle panels the host galaxy in the detection epoch and the rightmost panels the supernova candidate in the subtracted frame. The crosses in the leftmost panels mark the positions of the candidates. The candidate in the upper panel has an $I$ band magnitude of 22.7 and in the lower panel 24.8. See text for more details.

use of stamps, it is important that there is a sufficient number of sources left without artificial supernovae. The image subtraction process should, however, be able to deal with the presence of a supernova inside a previously selected stamp (and reject it from the stamp list). In a real survey it is very unlikely that more than one supernova will be found in the same galaxy during the same survey period. The number of SNe per galaxy has therefore been limited to one.

With these considerations in mind we are selecting host galaxies at random from a galaxy list, which, depending on the specific test run, can contain different samples of the full galaxy catalogue. The positions of the artificial sources inside the host galaxies are selected based on the flux profile for the specific host galaxy (in the general case, some test runs use a slightly different setup). Four position bins were used, where the in- and outside radii of the bins are given by: 0 to $r_{20} ; r_{20}$ to $r_{40} ; r_{40}$ to $r_{60} ; r_{60}$ to $r_{80}$. The angle with respect to the major axis of the galaxy and the radius within each bin were randomly selected. A fifth "position bin" was also considered, namely isolated positions, i.e. positions in the image without any detected galaxy within a given isolation radius. These positions were found by masking the detected sources in the reference image, using a $5 \sigma$ cutting threshold, dividing the resulting image into rectangular boxes with half-sides corresponding to the isolation radius. For the SVISS data an isolation radius of 20 pixels $\sim 4^{\prime \prime}$ was used. The wanted number of supernova positions were then selected at random from this list of boxes and sources placed in the middle of the boxes (one source per box).

In Fig. 2 an example of an image with artificial SNe is shown, together with the subtracted frame. The lower right panel of the figure shows the subtracted frame without any simulated sources. Typically, each subtracted frame will contain hundreds of residuals similar to the ones in this figure.

\subsection{Tests using the SVISS data set}

We have carried out a number of test runs with simulated supernovae added to the real images to study the effects on the subtraction quality and SN detection. A summary of the properties of the different runs is presented in Table 3 for the SVISS data set. The tests have been done using four different epochs (the A, B, C and D designations, see Table 2 for basic properties of the different epochs). The reference runs for each epoch, A0-D0, are tests using an identical set of parameters. For each epoch we have then changed one parameter at a time to study its effect on the subtraction quality. Some parameters have been studied in many epochs, while some have only been studied in the epoch 2 data. The same reference epoch (epoch 0 ) has been used for all the runs.

The stamp selection has been done in three different ways (e.g. A0, AS1 and AS2). In the "High SNR" option only the brightest sources in the field have been selected as stamps and in the "Auto" option the ISIS internal automatic stamp selection has been used. For the "Point" option, the most point-like sources have been selected as stamps. The total number of stamps in each of the three settings was $\sim 450$ over the entire frame (50 per subregion).

The tests on host galaxy brightness (i.e. 0, H1-H2) were done by selecting hosts from a magnitude limited galaxy list. In the $\mathrm{H} 1-\mathrm{H} 2$ runs the number of simulated SNe was a bit lower than in the reference runs, 100 instead of 266 . For the " $I \lesssim 22$ " option the 100 brightest sources were selected as hosts, the mean brightness of these galaxies was $I=20.0$. For the " $22 \lesssim I \lesssim 24$ " option 100 sources were selected at random from a magnitude limited catalogue, the mean brightness of these sources was $I=23.2$. The mean brightness of galaxies in the full host galaxy catalogue was $I=23.7$. In the case of the brightest host galaxies, some stamps had to be removed manually as they were not rejected by ISIS despite the addition of a bright SN to the stamp 


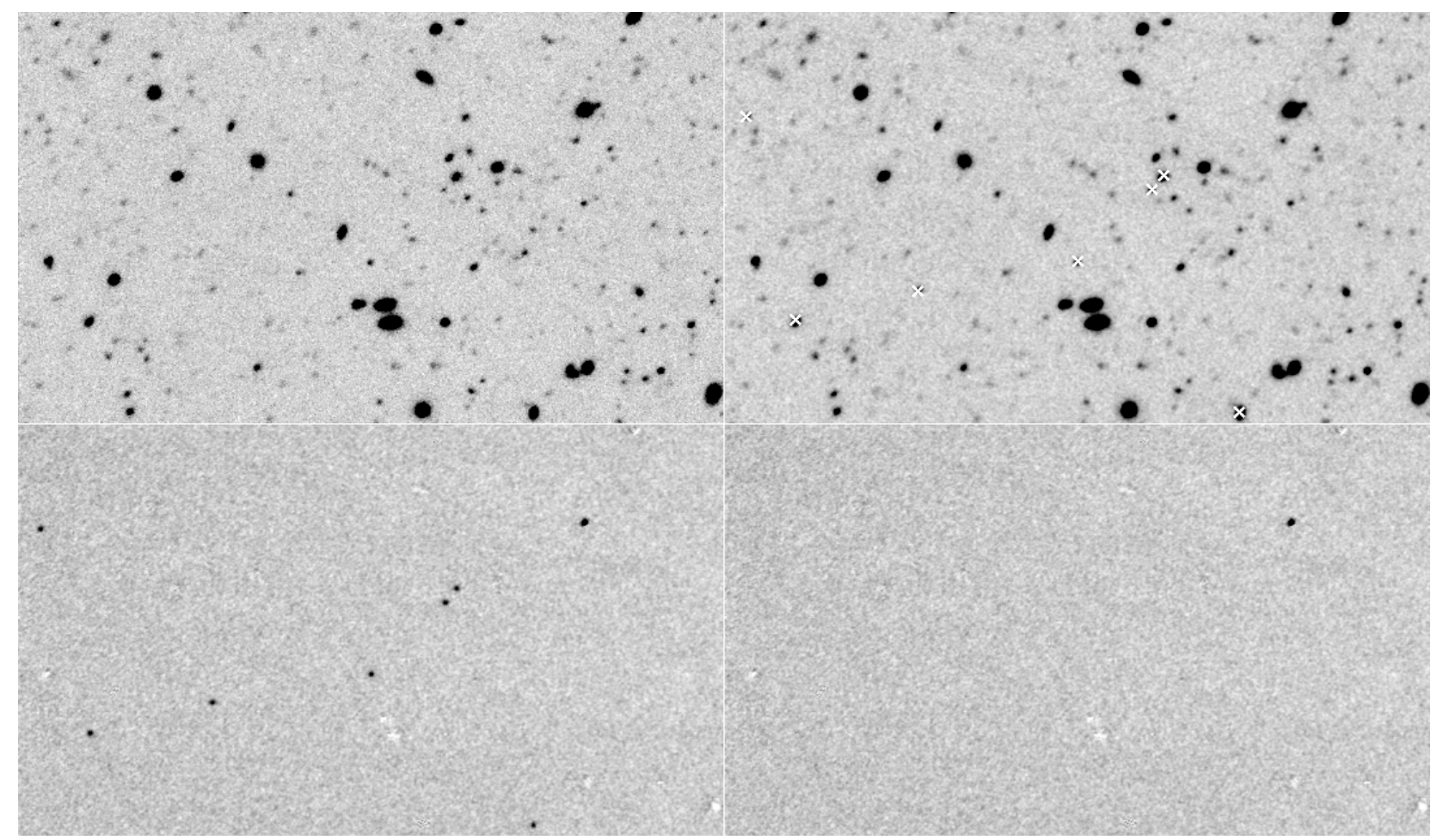

Fig. 2. An example image from the SVISS data set containing a number of simulated supernovae. The field of view in the image is $1.9^{\prime} \times 1.1^{\prime}$ (the total field of view of one VIMOS CCD is $\sim 7^{\prime} \times 8^{\prime}$ ). The top left frame is the reference image, the top right frame is the epoch 2 image with supernovae added (marked with with white crosses). The bottom left frame is the subtracted image (from run A0), the remaining sources are the added SNe. Some residuals are visible in the subtracted frame where no SNe have been added to the image (bottom right frame).

region. Having SN host galaxies as stamps affected the subtraction quality strongly for the brightest supernova bins.

In the W1-W5 runs, the width of the Gaussian basis functions that constitute the fitting kernel have been changed. The default ISIS values for the widths (expressed as the Gaussian $\sigma$ parameter) are $(1.0,2.0,4.0)$. In the test runs we are using both narrower and broader widths. The optimal kernel should contain a basis function with width that when convolved with the reference image will produce an image with the same seeing as the search image. For the SVISS images (see Table 2) the optimal kernel should thus contain a basis function with $\sigma \sim 0.4-0.8$.

\subsection{Test diagnostics}

\subsubsection{Pixel statistics in subtracted images}

In the AL98 and A00 papers pixel statistics of the subtracted images are used as a measure of the subtraction quality. The theoretical pixel noise distribution (hereafter PND) is compared to the measured distribution in the subtracted frames. The images used in these subtractions are simulated images with a purely Gaussian PND. The theoretical distribution is thus quite simple to calculate, even though convolution of the reference image makes it somewhat more complicated. In our tests we have used real data images with many sources, both point sources and extended objects, the PNDs for these images are harder to model. We have opted to take a more observationally orientated approach. Instead of comparing the PND in the subtracted image with a theoretical distribution, we compare the PND of isolated positions in the subtracted image to that of positions inside galaxies (see Fig. 3 for an example of this). The PND for isolated positions is thus considered to be the optimal one, the closer the
PND of the inside galaxy positions is to the isolated case, the more optimal the subtraction.

The pixel lists used in the statistics contain all pixels inside a $3^{\prime \prime}$ radius from all of the simulated $\mathrm{SN}$ positions, i.e. the pixel lists are obtained from a list of apertures, measured in an "empty" (without simulated SNe) subtracted frame. The positions for the isolated case are taken from the fifth (isolated) position bin and the inside galaxies positions from the $r 1$ position bin. The same number of apertures have been used for both pixel lists, so that the total number of pixels is the same within interpolation errors.

For each of the test runs we have calculated three different statistics based on comparing the PNDs: (i) the pixel to pixel noise inside the apertures of the inside positions and the isolated positions, $\sigma_{\mathrm{r} 1}$ and $\sigma_{\mathrm{r} 5}$; (ii) the reduced $\chi^{2}$ statistic $\chi_{\text {red }}^{2}$ computed from comparing the PNDs; (iii) the flux offset statistic, $\mathrm{d} f$.

The flux offset statistic is obtained by numerical integration of the PND difference over the counts per pixel and dividing by the number of apertures used. The flux offset is defined as:

$$
\begin{aligned}
\mathrm{d} f= & \frac{1}{n_{\mathrm{ap}}}\left(\int_{l l}^{0}-\left(P_{\mathrm{r} 1}(x)-P_{\mathrm{iso}}(x)\right) \mathrm{d} x\right. \\
& \left.+\int_{0}^{u l}\left(P_{\mathrm{r} 1}(x)-P_{\mathrm{iso}}(x)\right) \mathrm{d} x\right)
\end{aligned}
$$

where $n_{\mathrm{ap}}$ is the number of apertures, $l l$ and $u l$ are the upper and lower limit of the counts per pixel, respectively, $x$ counts per pixel and $P_{\mathrm{r} 1 / \text { iso }}$ the number of pixels in the interval $\mathrm{d} x$ for a given value of $x$. The $\mathrm{r} 1$ and iso designations refer to the two position bins considered (see above). This statistic is in principle the mean counts per aperture with the background, defined as the counts inside the isolated position apertures, removed. The 
Table 3. Properties and results of the test runs using the SVISS data set.

\begin{tabular}{|c|c|c|c|c|c|c|c|}
\hline Run & Stamp selection & Host brightness & Kernel widths & $\sigma_{\mathrm{r} 5} / \sigma_{\mathrm{r} 1}$ & $\chi_{\text {norm }}^{2}$ & $\mathrm{~d} f$ & $r_{\mathrm{sp}}$ \\
\hline A0 & High SNR & $I \lesssim 25$ & $(1.0,2.0,4.0)$ & $0.026 / 0.034$ & 1.00 & 0.13 & 0.825 \\
\hline AS1 & Auto & $I \lesssim 25$ & $(1.0,2.0,4.0)$ & $0.024 / 0.031$ & 2.97 & 0.22 & 0.848 \\
\hline AS2 & Point & $I \lesssim 25$ & $(1.0,2.0,4.0)$ & $0.024 / 0.034$ & 2.87 & 0.12 & 0.891 \\
\hline AH1 & High SNR† & $I \lesssim 22$ & $(1.0,2.0,4.0)$ & $0.027 / 0.077$ & 7.34 & 0.47 & 0.825 \\
\hline $\mathrm{AH} 2$ & High SNR & $22 \lesssim I \lessgtr 24$ & $(1.0,2.0,4.0)$ & $0.027 / 0.038$ & 1.76 & 0.27 & 0.825 \\
\hline AW1 & High SNR & $I \lesssim 25$ & $(0.2,0.4,0.8)$ & $0.027 / 0.033$ & 1.39 & 0.13 & 0.831 \\
\hline AW2 & High SNR & $I \lesssim 25$ & $(0.4,0.8,1.6)$ & $0.026 / 0.033$ & 0.924 & 0.13 & 0.821 \\
\hline AW3 & High SNR & $I \lesssim 25$ & $(0.6,1.2,2.4)$ & $0.026 / 0.032$ & 0.855 & 0.10 & 0.816 \\
\hline AW4 & High SNR & $I \lesssim 25$ & $(2.0,4.0,8.0)$ & $0.024 / 0.034$ & 1.88 & 0.11 & 0.834 \\
\hline AW5 & High SNR & $I \lesssim 25$ & $(4.0,8.0,16.0)$ & $0.027 / 0.035$ & 1.02 & 0.14 & 0.826 \\
\hline B0 & High SNR & $I \lesssim 25$ & $(1.0,2.0,4.0)$ & $0.023 / 0.035$ & 1.00 & -0.19 & 0.667 \\
\hline BS1 & Auto & $I \lesssim 25$ & $(1.0,2.0,4.0)$ & $0.025 / 0.034$ & 1.25 & -0.32 & 0.726 \\
\hline BS2 & Point & $I \lesssim 25$ & $(1.0,2.0,4.0)$ & $0.026 / 0.036$ & 1.34 & -0.29 & 0.808 \\
\hline BH1 & High SNR† & $I \lesssim 22$ & $(1.0,2.0,4.0)$ & $0.024 / 0.084$ & 2.91 & -0.088 & 0.667 \\
\hline $\mathrm{BH} 2$ & High SNR & $22 \lessgtr I \lessgtr 24$ & $(1.0,2.0,4.0)$ & $0.024 / 0.041$ & 1.06 & -0.28 & 0.667 \\
\hline BW1 & High SNR & $I \lesssim 25$ & $(0.2,0.4,0.8)$ & $0.024 / 0.034$ & 0.638 & -0.030 & 0.648 \\
\hline BW2 & High SNR & $I \lesssim 25$ & $(0.4,0.8,1.6)$ & $0.023 / 0.033$ & 0.823 & -0.13 & 0.659 \\
\hline BW3 & High SNR & $I \lesssim 25$ & $(0.6,1.2,2.4)$ & $0.023 / 0.033$ & 1.04 & -0.20 & 0.672 \\
\hline BW4 & High SNR & $I \lesssim 25$ & $(2.0,4.0,8.0)$ & $0.024 / 0.035$ & 1.06 & -0.22 & 0.677 \\
\hline BW5 & High SNR & $I \lesssim 25$ & $(4.0,8.0,16.0)$ & $0.026 / 0.046$ & 0.672 & -0.14 & 0.661 \\
\hline $\mathrm{CO}$ & $\begin{array}{l}\text { High SNR } \\
\text { Sigh }\end{array}$ & $I \lesssim 25$ & $(1.0,2.0,4.0)$ & $0.026 / 0.034$ & 1.00 & -0.21 & 0.686 \\
\hline $\mathrm{CS} 1$ & Auto & $I \lesssim 25$ & $(1.0,2.0,4.0)$ & $0.027 / 0.035$ & 0.83 & -0.18 & 0.674 \\
\hline $\mathrm{CS} 2$ & Point & $I \lesssim 25$ & $(1.0,2.0,4.0)$ & $0.032 / 0.041$ & 1.32 & -0.25 & 0.808 \\
\hline $\mathrm{CH} 1$ & High SNR $\dagger$ & $I \lesssim 22$ & $(1.0,2.0,4.0)$ & $0.028 / 0.070$ & 2.92 & -0.69 & 0.686 \\
\hline $\mathrm{CH} 2$ & High SNR & $22 \lessgtr I \lessgtr 24$ & $(1.0,2.0,4.0)$ & $0.028 / 0.039$ & 1.40 & -0.51 & 0.686 \\
\hline CW1 & High SNR & $I \lesssim 25$ & $(0.2,0.4,0.8)$ & $0.026 / 0.032$ & 0.921 & -0.20 & 0.674 \\
\hline CW2 & High SNR & $I \lesssim 25$ & $(0.4,0.8,1.6)$ & $0.026 / 0.034$ & 1.19 & -0.25 & 0.693 \\
\hline CW3 & High SNR & $I \lesssim 25$ & $(0.6,1.2,2.4)$ & $0.026 / 0.035$ & 0.949 & -0.20 & 0.685 \\
\hline CW4 & High SNR & $I \lesssim 25$ & $(2.0,4.0,8.0)$ & $0.026 / 0.034$ & 1.06 & -0.20 & 0.688 \\
\hline CW5 & High SNR & $I \lesssim 25$ & $(4.0,8.0,16.0)$ & $0.026 / 0.036$ & 1.18 & -0.21 & 0.701 \\
\hline D0 & High SNR & $I \lesssim 25$ & $(1.0,2.0,4.0)$ & $0.025 / 0.033$ & 1.00 & -0.0053 & 0.734 \\
\hline DS1 & Auto & $I \lesssim 25$ & $(1.0,2.0,4.0)$ & $0.025 / 0.034$ & 1.19 & 0.048 & 0.737 \\
\hline DS2 & Point & $I \lesssim 25$ & $(1.0,2.0,4.0)$ & $0.029 / 0.039$ & 1.19 & 0.022 & 0.832 \\
\hline DH1 & High SNR $\dagger$ & $I \lessgtr 22$ & $(1.0,2.0,4.0)$ & $0.025 / 0.069$ & 4.00 & 0.21 & 0.734 \\
\hline DH2 & High SNR & $22 \lesssim I \lesssim 24$ & $(1.0,2.0,4.0)$ & $0.027 / 0.038$ & 1.10 & 0.099 & 0.734 \\
\hline DW1 & High SNR & $I \lesssim 25$ & $(0.2,0.4,0.8)$ & $0.072 / 0.061$ & 2.15 & 0.10 & 0.821 \\
\hline DW2 & High SNR & $I \lesssim 25$ & $(0.4,0.8,1.6)$ & $0.030 / 0.036$ & 1.06 & 0.033 & 0.738 \\
\hline DW3 & High SNR & $I \lesssim 25$ & $(0.6,1.2,2.4)$ & $0.028 / 0.036$ & 1.18 & 0.071 & 0.738 \\
\hline DW4 & High SNR & $I \lesssim 25$ & $(2.0,4.0,8.0)$ & $0.029 / 0.040$ & 1.27 & 0.079 & 0.741 \\
\hline DW5 & High SNR & $I \lesssim 25$ & $(4.0,8.0,16.0)$ & $0.028 / 0.040$ & 1.66 & 0.044 & 0.749 \\
\hline
\end{tabular}

$\sigma_{\mathrm{r} 5}$ and $\sigma_{\mathrm{r} 1}$ is the pixel to pixel noise in the isolated and inside galaxies positions, respectively. $\chi_{\text {norm }}^{2}$ is the normalised $\chi^{2}$ statistic for the run, $\mathrm{d} f$ is the flux difference statistic and $r_{\mathrm{sp}}$ is the spurious detection ratio (see Sect. 4.4.1 for details).

$\dagger$ Bright sources with simulated SNe have been manually removed from the stamp list.

numerical integration of the PND difference enables us to control the lower and higher bounds as well as making sure that the correct background, i.e. the counts within isolated position apertures, is removed.

\subsubsection{Detection efficiency}

For each of the magnitude bins a number of detected sources is obtained from the SE runs. A detected source is defined as having a signal-to-noise ratio $(S N R)$ of more than one, "sources" detected by SE can have very low significance and include many spurious detections. The input simulation coordinate list is then used as an ASSOC list in SE (i.e. matched in image coordinates) to make sure that only simulated objects are counted in the detection. In this way we can study the detection efficiency for a certain set of parameters in real data without including spurious detections from residual sources. This estimate of the survey depth is useful when other methods can be used to dispose spurious detections, e.g. by using multiple bands or epochs. For single epoch measurements greater care has to be taken not to include spurious objects.

The detection efficiency is then calculated for each magnitude bin as the number of detected sources divided by the total number of simulated objects in the magnitude bin. However, these will not be the true detection efficiencies since the SNR estimated by SE is not accurate due to the correlated pixel to pixel noise in the subtracted images. Such detection efficiencies would thus be unreliable to use in the $\mathrm{SN}$ rate calculations, and in this paper we use them only for comparison between the different subtraction parameters and epochs. Due to the way we handle detections in the images with simulated $\mathrm{SNe}$ (only accepting detections at positions where $\mathrm{SNe}$ were placed), the signal to noise cut used does not affect the results discussed in this paper. For the $\mathrm{SN}$ rate calculations we will use detection efficiencies based on signal to noise cuts found from doing PSF photometry of simulated $\mathrm{SNe}$ (using the photometric scatter method described below). 


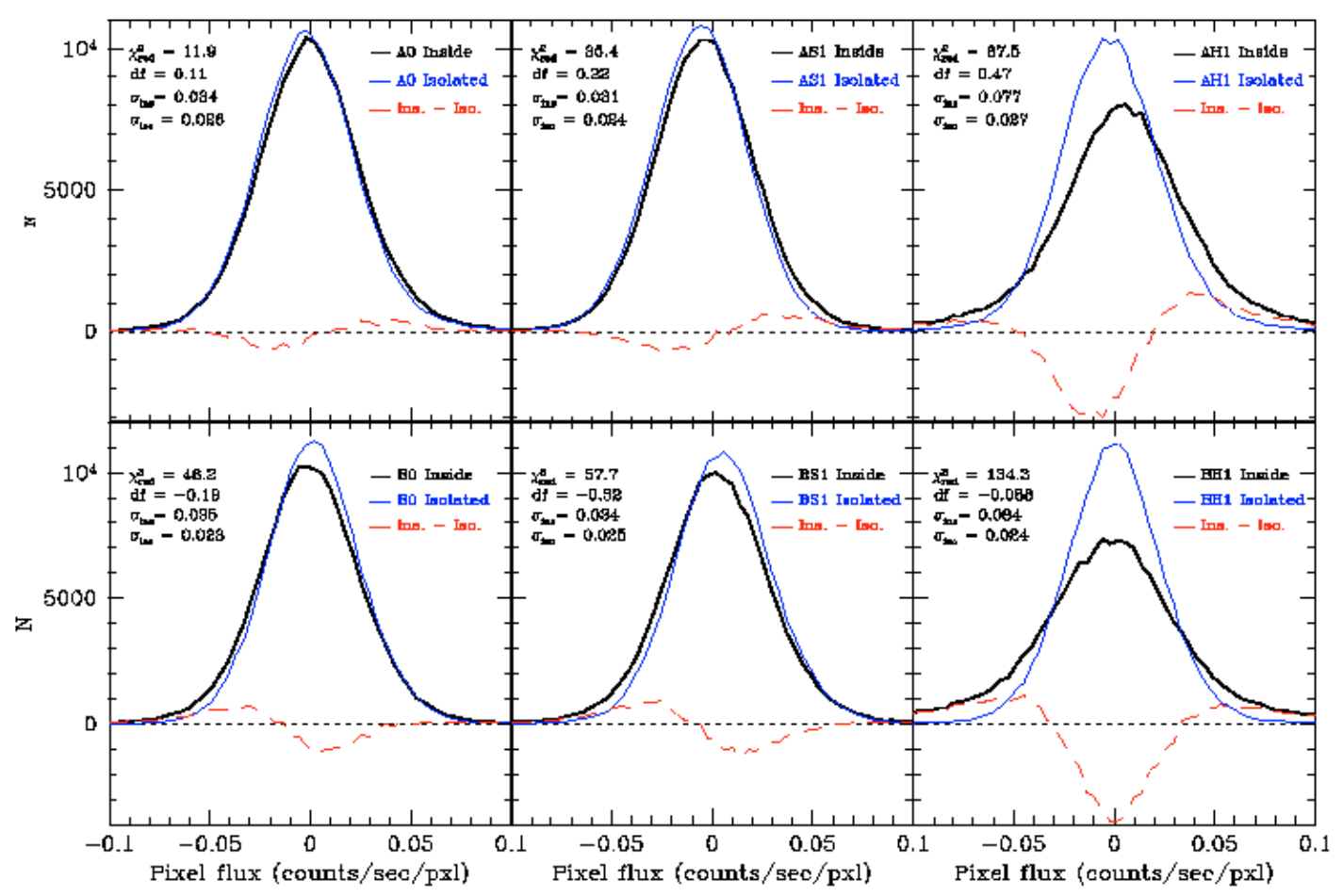

Fig. 3. Pixel noise distributions (PNDs) for two of the search epochs (upper panel shows search epoch A, lower epoch B). Leftmost panels show the reference test runs (using high-SNR stamps and completely random host galaxies), middle panels show the S1 runs (using ISIS automatic stamp selection) and rightmost panels show H1 runs (bright host galaxies). All distributions, except the bright host galaxy test, contain the same total number of pixels (for details see Table 1 and Sect. 4.4.1). The dashed (red) line show the difference of the inside and outside pixel distributions for each case.

\subsubsection{Photometric accuracy}

The photometry of the supernova candidates is done in the subtracted images using PSF photometry (see Sect. 2.4). The accuracy of this method has been assessed by subtracting the input simulation magnitudes from the measured magnitudes for each of the simulated sources and then calculating the mean, median and standard deviation of the magnitude differences. The magnitudes have also been converted to flux (in counts/s) using the magnitude zeropoints. The difference in flux and magnitude versus input simulation magnitude have then been plotted for the different cases. It is important to note that each of the magnitude bins is independent of each other, the host galaxy and simulated SN position are selected at random for each of the bins. Any offset in the the accuracy plots thus means that the entire image/method suffers from a systematic error, rather than effects from selecting a "bad" sample. Tests have also been done using aperture photometry with aperture correction, this gives very similar results but with a larger scatter.

The standard deviation of detected $\mathrm{SNe}$ in each magnitude bin is used as an estimate of the photometric scatter. As discussed in Sect. 2.4 the error estimates from daophot and phot will not give accurate photometric errors. The standard deviation in each simulated magnitude bin should provide a more accurate estimate for the statistical photometric errors. The $S N R$ for a given magnitude is computed from the magnitude scatter using the formula $S N R \approx 1.0857 \times \frac{1}{\sigma_{\text {mag }}}$.

A problem with estimating photometric accuracy in this way is that the detected sample will be incomplete at the faintest magnitudes. Only the very brightest sources in a given magnitude bin (i.e. sources that happen to be located at the position of a noise peak or residual flux peak) will be detected at the faint limit. This can be seen in the photometric accuracy plots, e.g. Fig. 5, where the flux difference becomes positive (indicating that the flux is overestimated) in the faintest magnitude bins. Conclusions should only be drawn based on the complete bins.

\subsubsection{Spurious detections}

Whenever source detection codes are used to do automatic detections there is a risk that some of the detected sources are in fact not real, but rather noise peaks that are mistaken for real objects. The number of spurious detections is affected by the detection parameters, a very conservative setting of these parameters can result in few spurious detections. However, the problem with using conservative detection parameters is that real, but faint, sources might be missed. In galaxy surveys it is important that the number of spurious detections is known and, in some cases, controlled. Different methods can be used to do this, one is to run the detection again with the same parameters on the inverted image. If the noise is Gaussian the amount of spurious detections is expected to be similar in both the images.

For the subtracted images used in this work this method is not suitable. Many of the spurious detections will not be related to the Gaussian noise in the isolated parts of the image, but rather associated with residuals from galaxies that were not fully subtracted. The "noise" in these residuals is not necessarily Gaussian nor symmetric, thus the inverted image is not a good tool to use.

We instead define a spurious detection in the subtracted images as a source that is detected in the test search epoch $I$ band images, but not detected in the $R$ band images for the same epoch. The spurious detection ratio is thus defined as:

$r_{\mathrm{sp}}=\frac{n_{I}-n_{I \cap R}}{n_{I}}$ 


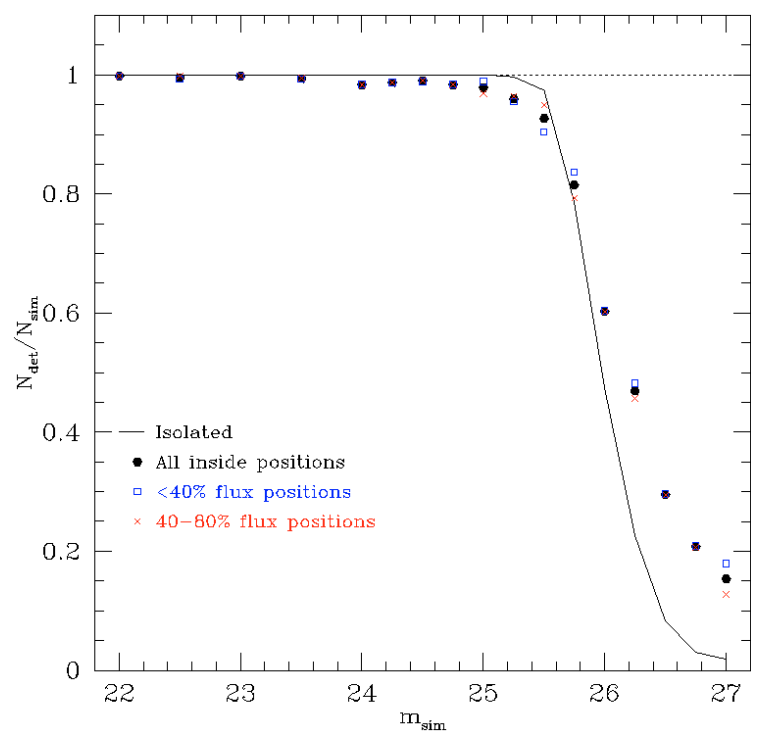

Fig. 4. Detection efficiency for isolated positions and different galactocentric distances inside the host galaxies. The plot was constructed from the A0 run.

where $n_{I}$ is the number of detected sources in the $I$ band image, which is the filter used for all of the tests. $n_{I \cap R}$ is the number of sources detected in both $I$ and $R$. Source detection using SE is done in "empty" subtracted images for the different parameter sets. For this test the detected sources are from the entire image, not only from the simulated positions considered for the detection efficiency tests.

\section{Results}

Our tests show that the $\sigma_{\mathrm{r} 1} / \sigma_{\mathrm{r} 5}, \chi_{\text {red }}^{2}$ and $\mathrm{d} f$ statistics can be used as measures on how successful the difference imaging was in subtracting galaxies from positions where artificial SNe were added. We have also normalised the $\chi_{\text {red }}^{2}$ statistic for each test run by the value found for the reference run (A0, B0, C0 and D0). The normalised $\chi_{\text {red }}^{2}$ makes it easier to compare the test runs within each epoch, but can not be used to compare epochs to each other. Table 3 contains the resulting statistics for the test runs, and Fig. 3 shows the pixel noise distributions for some of them.

Some general remarks can be made on the relationship between the different statistical measures. By definition $\chi_{\text {red }}^{2}$ will be a measure of the total difference of the two distributions. There are two main components to this total difference, one is the difference in widths of the two distributions (characterised by the standard deviations, $\left.\sigma_{\mathrm{r} 1} / \sigma_{\mathrm{r} 5}\right)$. The $\mathrm{d} f$ statistic is mainly sensitive to possible systematic flux offsets, which is the other main component in the $\chi_{\text {red }}^{2}$, and gives an indication on whether the offset is positive or negative. In Table 3 we see that, as expected, a large $\sigma_{\mathrm{r} 1}$ and/or a large $\mathrm{d} f$ (negative or positive) results in a large $\chi_{\text {red }}^{2}$. This is also evident from looking at the pixel distribution plots in Fig. 3.

\subsection{Detection and photometry of supernovae with faint host galaxies}

To test the effects of the supernova galactocentric distance we placed the simulated sources as described in Sect. 4.2. The resulting detection efficiency and photometric accuracy for the different position bins are shown in Figs. 4 and 5. While the position tests have been done in all the test epochs, these figures show only run $\mathrm{A} 0$. The four position bins inside each galaxy were merged into two, one region containing $40 \%$ of the flux and the other one containing 40 to $80 \%$ of the flux. We find no difference within the errors between the inside position bins, neither for detection efficiency nor photometric accuracy. The small differences visible in the figures is smaller than the scatter for each magnitude bin. The scatter in the detection efficiency plot is hard to calculate, but the fact that for values for the two positions switch order between magnitude bins can be interpreted as a scatter effect. This result holds true for all of the test runs.

The resulting efficiency and photometric accuracy for the isolated positions differs significantly from the ones for inside positions. Assuming the isolated SN positions to be an ideal case and comparing the photometric accuracy for these positions with the inside ones, we find that there is a systematic error in the photometry for the inside positions. Further testing showed that this systematic error is in fact a flux offset, the difference between simulated and measured flux is the same in all of the magnitude bins (shown in the left panels of Figs. 5 and 7). In the case of run A0 the flux offset is positive, positions inside galaxies have too much flux remaining after subtraction. This is also visible in the detection efficiency plot, the efficiency for inside positions is erroneously boosted by the extra flux, giving an efficiency better than the isolated case, which is clearly incorrect. The error bars given in the flux difference panels show the standard error of mean in each bin. The difference is nonzero (within 3-5 $\sigma$ ) and is consistent with a constant flux offset in all of the magnitude bins. Using the mean flux of the faint host galaxy sample and the measured flux offset we can calculate the mean fraction of host flux that remains in the subtracted frame. For the A, $\mathrm{B}, \mathrm{C}$ and $\mathrm{D}$ epochs the fraction is $1.9 \%, 3.0 \%, 2.5 \%$ and $5.3 \%$, respectively.

The subtraction has succeeded to varying degree in the four different search epochs, the detection efficiency and photometric accuracy for the reference runs of each epoch is shown in Figs. 6 and 7, as well as in Table 3. The $\chi_{\text {red }}^{2}$ statistic for the four reference runs are in fact very different, 11.9, 46.2, 32.7 and 17.3, respectively, for A0-D0. This is not surprising since the data from the different epochs are of somewhat differing quality (see Table 2). The flux offset has been detected in all of the test epochs to varying degree, in epoch $\mathrm{A}$ it is a positive offset and in B, C and D a negative one. This is clearly visible both in the trend for the $\mathrm{d} f$ statistic and in the photometric accuracy plot (Fig. 6). In all of the epochs, a flux offset also results in an artificial boost/suppression of the detection efficiency.

The lower panels of Figs. 5 and 7 show the SNR and standard deviation of the measured magnitudes in each bin for the different positions and epochs. Contrary to the standard error of mean, the scatter is an estimate of the photometric uncertainty for individual supernovae. As expected, the scatter is considerably smaller in the isolated positions compared to the inside positions. The scatter and $S N R$ for the three epochs are very similar, but the detection efficiency is different. This shows that the detection efficiency is affected by the systematic flux offset. The SNR for a given simulated magnitude bin is higher for the isolated positions than for the inside positions (lower left panel of Fig. 5). This is of course as expected since the presence of a host galaxy will affect the photometry of the SN. The estimates of SNR and scatter are unreliable for the faintest magnitude bins since the sample becomes incomplete in these bins (e.g., see Fig. 4). A useful estimate of the depth can be found by extrapolating from the trustworthy data points where the detections are $100 \%$ complete. The $3 \sigma$ detection limit for the isolated 


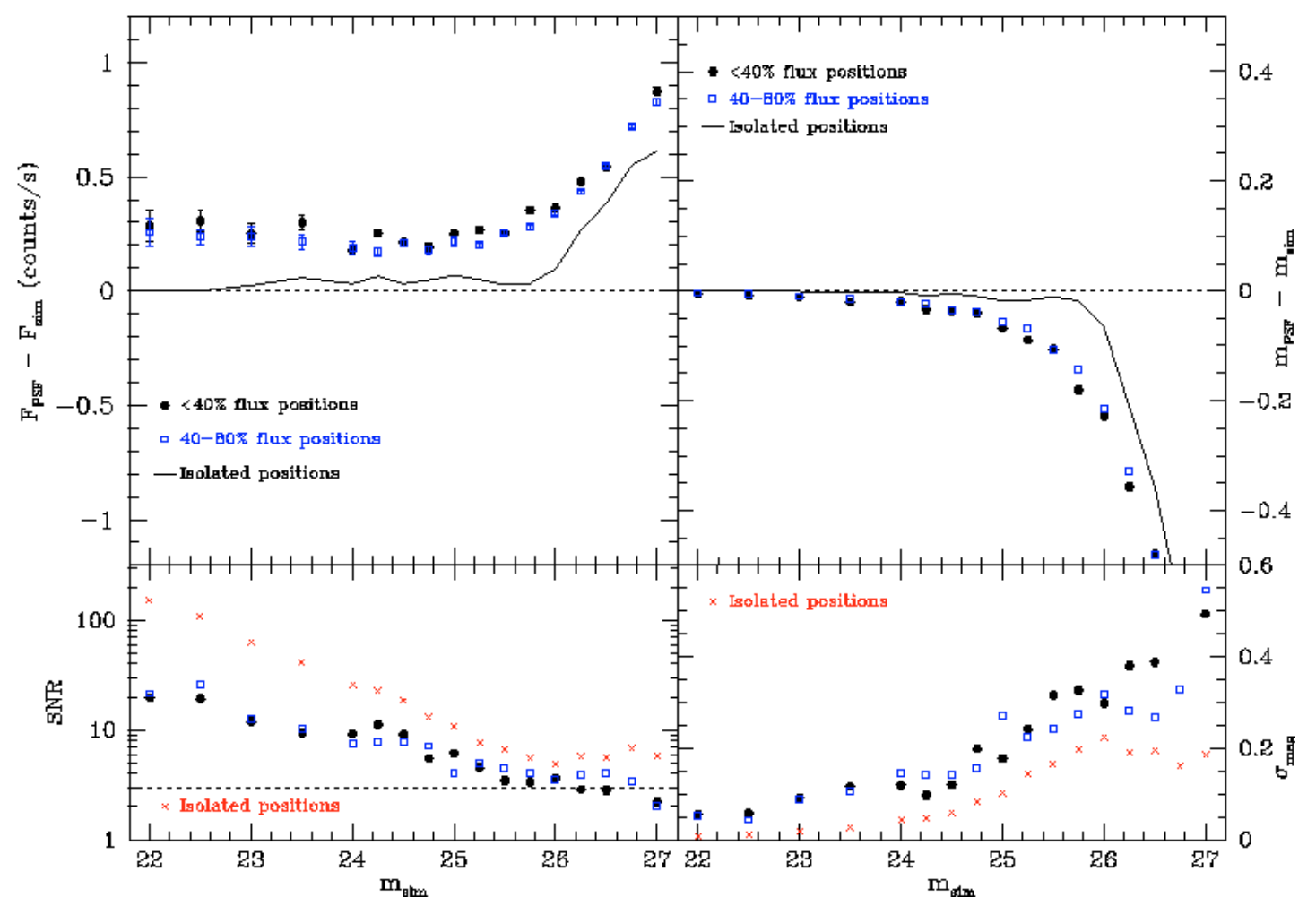

Fig. 5. Photometric accuracy for $\mathrm{SNe}$ at different galactocentric distances as a function of simulation magnitude $\left(m_{\text {sim }}\right)$. The plot was constructed from the $\mathrm{A} 0$ run. The upper left panel shows the median pixel flux difference (in counts/s) between the input simulation flux $\left(F_{\text {sim }}\right)$ and the measured PSF flux $\left(F_{\mathrm{PSF}}\right)$, and the upper right panel shows the median magnitude difference. Error bars in the flux difference plot show the standard error of mean of the difference. Note that the detections become incomplete at magnitudes $\gtrsim 26$, this causes the apparent failure of photometry at those faint magnitudes. The lower left panel shows the signal to noise versus the simulation magnitude and the lower right panel the measured scatter in each simulation bin. The signal to noise ratios have been estimated from the measured scatter $\left(S N R \approx 1.0857 \times \frac{1}{\sigma_{\mathrm{mag}}}\right)$. The dashed horizontal line marks the $S N R=3$ limit.

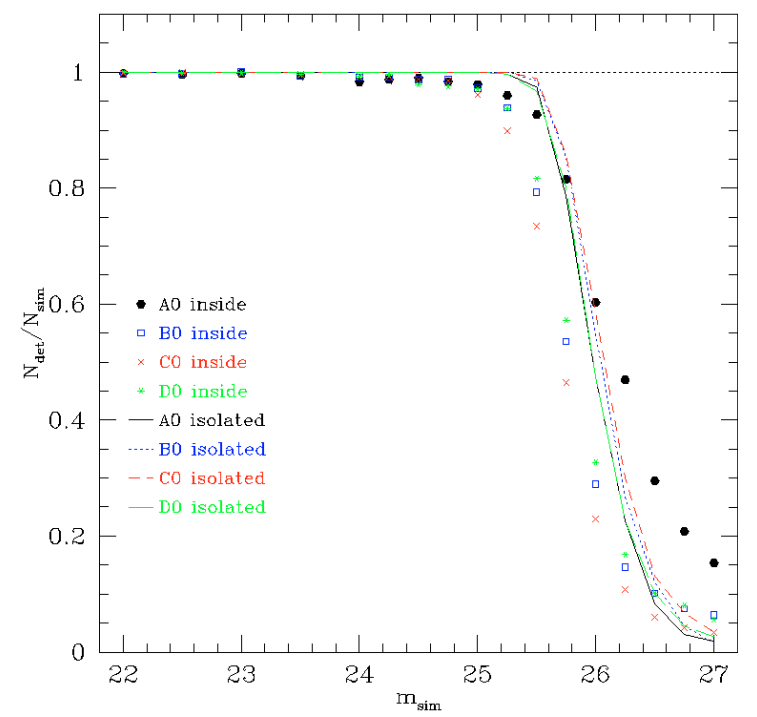

Fig. 6. Detection efficiencies for isolated and inside galaxies positions at the four different search epochs.

position is approximately $m_{I}=26.1$. This is comparable to the $3 \sigma$ limiting magnitude for the individual frames (see Table 2). The increase in noise from subtracting the images which should cause the limiting magnitudes in the subtracted images to be on the order of $0.4 \mathrm{mag}$ lower is not seen. This is due to the effects discussed in Sect. 2.4, where the subtraction actually serves to remove the problematic background, this effect is roughly similar (by coincidence) to the increase in pixel to pixel noise. The $S N R$ versus magnitude plots for the four different epoch (lower left panel of Fig. 7) show that the B epoch is considerably less deep than the other three epochs. This is in agreement with the limiting magnitudes given in Table 2, where epoch B is indeed the shallowest of the four epochs. The $50 \%$ completeness limit roughly corresponds to the $3 \sigma$ limiting magnitude obtained from the SNR plots, but it should be noted that the flux offsets have a fairly strong effect on the detection efficiencies. The number of spurious detections, characterised by $r_{\mathrm{sp}}$, also reflects this, the number is significantly higher in the A epoch (which has a positive flux offset) than in the epochs with negative offsets.

\subsection{Detection and photometry of supernovae with bright host galaxies}

Properties of the runs with different host galaxy characteristics can be found in Table 3 (H1 and $\mathrm{H} 2$ runs for the 4 different search epochs). Figures 8 and 9 show the detection efficiency and photometric accuracy in the reference, $\mathrm{H} 1$ and $\mathrm{H} 2$ runs for one of the epochs (epoch A).

The detection efficiency plot clearly shows that the efficiency becomes worse when the host galaxy is considerably brighter than the simulated supernovae. This could possibly be explained by the Poisson noise from the host galaxy eventually becoming dominating when fainter SNe are detected. Assuming that the noise for a source consists only of Poisson noise from the host galaxy, i.e. $S N R \sim F_{\mathrm{SN}} / \sqrt{F_{\text {host }}}$, we find that the SN magnitude for a $90 \%$ detection efficiency corresponds to a 


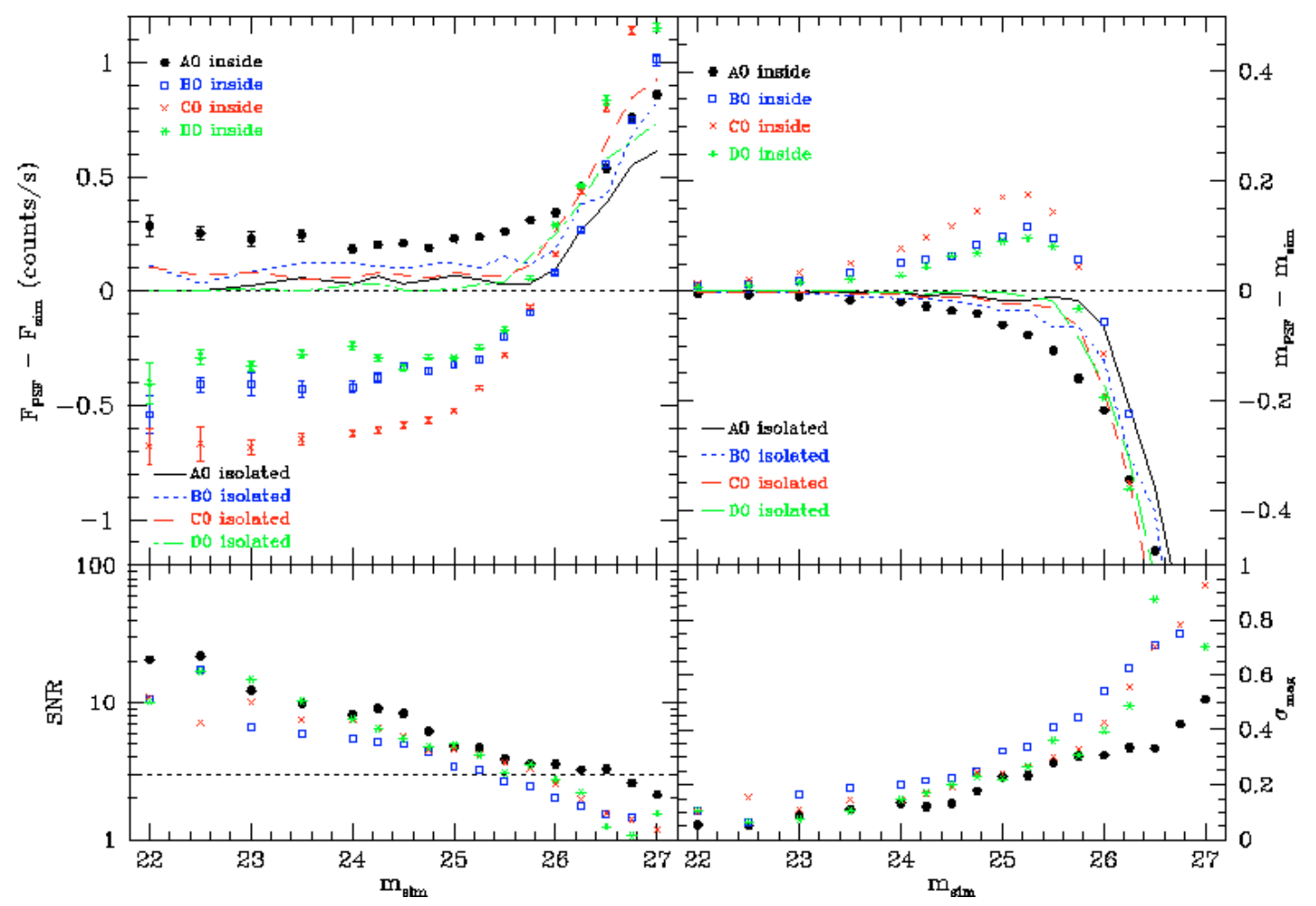

Fig. 7. Photometric accuracy for the different search epochs. Panels are the same as in Fig. 5. The legend shows the symbols used for the epochs.

signal-to-noise ratio of $\gtrsim 100$ for the bright host galaxy group. Thus it seems unlikely that the host galaxy Poisson noise affects the detection probability nor the photometry for the simulated SNe. Also, the drop at the detection limit is considerably slower for the bright and intermediate brightness hosts. These two effects can be explained as subtraction residuals from the host galaxies being detected as SNe. Visual inspection of the subtracted frames also show that almost all of the selected bright host galaxies show significant residuals. The pixel-to-pixel noise in apertures placed on the inside galaxies position $\left(\sigma_{r 1}\right.$ given in Table 3) is also increased substantially when considering brighter hosts. This is due to the subtraction residuals in these positions since the Poisson noise from nearly all but the very brightest galaxies is negligible in our deep and sky noise dominated images.

The photometric accuracy is also affected by the brightness of the host galaxies. The constant flux offset discussed in the previous section is no longer seen for the two populations of brighter host galaxies (see Fig. 9). In the very brightest galaxy population we see that the flux and magnitude differences are very large. There seems to be some kind of systematic offset inside the bright galaxies but it is no longer constant in the different magnitude bins. For this host sample, the magnitude bins are not independent of each other, the host galaxies are the same in all the bins, i.e. the 100 brightest galaxies in the field. The flux difference is in this case not independent of the SN flux. For the very faintest bins the difference is positive (which also the $\mathrm{d} f$ statistic for run AH1 in Table 3 indicates), while for the very brightest simulation bins the difference is negative. However, it should be noted that the scatter is very large for the bright hosts. The SNR plot for different host galaxy brightness (lower left panel of Fig. 9) shows that the $3 \sigma$ limiting magnitudes for the bright and intermediate host galaxy samples are a lot worse than for the faint galaxy sample.

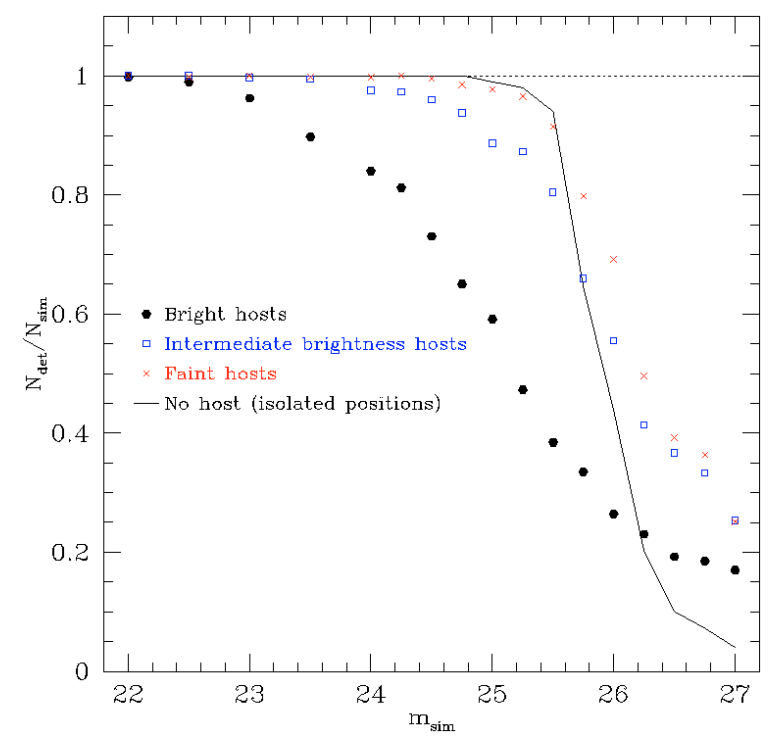

Fig. 8. Detection efficiency for the runs with different host galaxy brightness for a single epoch (A0, AH1-AH2). The mean $I$ band magnitudes for the three host galaxy populations are 20.0, 23.2 and 23.7.

A possible explanation for this effect could be that the photometry fails in the presence of powerful negative residuals, giving unpredictable results for the bright simulation bins. The intermediate brightness hosts are somewhere in between the faint and bright hosts, for the epoch A case they do not show any offset at all for any of the magnitude bins. We cannot find any simple relation between the host brightness and the found photometric difference, the epochs are affected differently, cf. AH-DH runs in Table 3 (Fig. 8 shows only epoch A). Since the subtraction residuals at the bright galaxy locations vary 


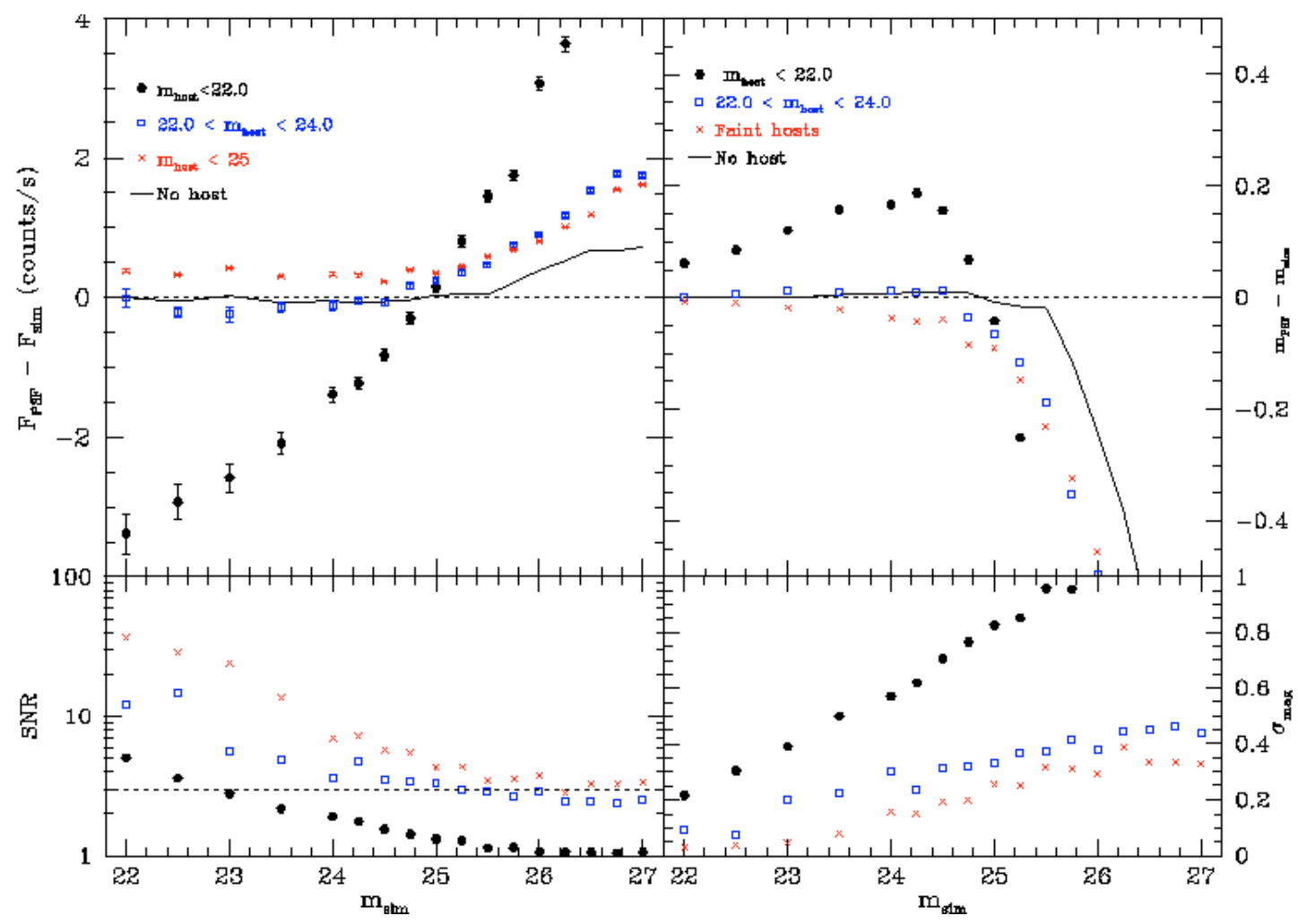

Fig. 9. Photometric accuracy for runs with different host galaxy brightness for a single epoch (A0, AH1-AH2). Panels are the same as in Fig. 5. The legends show the symbols used for the different host galaxy tests.

considerably from epoch to epoch, this is expected if the residuals is the source of the found photometric errors for bright host galaxies.

It should be noted that the bright host runs were carried out with a slightly modified stamp list. We found that bright galaxies with simulated supernovae were not correctly rejected by ISIS in the subtraction. The subtraction quality was therefore greatly degraded for the brighter magnitude bins. We manually removed the bright host galaxies with simulated SNe from the stamp list. The stamp rejection is further discussed in Sect. 5.4.

\subsection{Effects of convolution kernel properties}

Detailed testing on subtraction parameters in OIS methods have already been reported by, e.g., A00 and Israel et al. (2007). In these papers the detailed testing is done on fully simulated data where noise and subtraction residuals can be controlled. We have done some tests on varying the subtraction parameters in our real data, i.e., observed images with simulated SNe. In general, the noise and residuals from the host galaxies dominate over any effects resulting from subtraction parameter changes. We have therefore chosen to use the default ISIS 2.2 parameters for all parameters but the convolution kernel width (see Table 1). In the reference runs (the 0 runs) the default kernel widths have been used.

Israel et al. (2007) find that the widths of the basis functions of the kernel affect the subtraction result quite strongly. We have tested this in our real data and the results are shown in Table 3. The tests show that the effect of changing the widths is small for most of the tested width combinations. The $\chi_{\text {norm }}^{2}$ statistic indicate that the widest (W4-W5) kernel widths are the worst. The best kernel width parameter setting is different for the four epochs but in many cases kernel width combinations that are more narrow than the default give a better result. We have also calculated the detection efficiencies for several kernel width combinations. The 0 and $\mathrm{W} 1-\mathrm{W} 3$ runs all have very similar efficiencies, but for the widest width combinations the depth goes down considerably. This is consistent with the noise in the subtracted image $\left(\sigma_{r 1}\right)$ being $\sim 4$ times higher when using the widest basis functions (W4-W5) than in the reference case. In one case (BW5) the $\chi_{\text {norm }}^{2}$ statistic indicates that this is the best setting for that particular epoch, but the noise in the subtracted frame is high and the detection efficiency is very poor. For our data set, where the seeing differences are quite small, it is likely that the lack of at least one narrow basis function makes the convolution kernel suboptimal. In principle one would expect the pixel to pixel noise to go down when using a wider convolution kernel, but it seems that the higher subtraction residuals in reality increase the noise substantially in isolated positions and slightly in the non-isolated positions.

\subsection{Stamp selection}

The different stamp selection methods are compared using the subtraction quality diagnostic statistics in Table 1. The tests show that the "High SNR" option works best, both in terms of overall subtraction quality, as estimated by the $\chi_{\text {norm }}^{2}$ and $\mathrm{d} f$ diagnostics, and in terms of spurious detections. The "Point" option gives the worst results for all of the tested epochs. The "Auto" option gives differing results, which is not surprising since the stamp list will be remade for each epoch, in contrast to the two other methods where roughly the same stamps are used for all test epochs. In general, this method seems to give slightly worse results than the "High SNR" one.

It is also worth noting that the stamp rejection routine does not seem to work properly. Galaxies with $\mathrm{SNe}$ (bright or faint) 


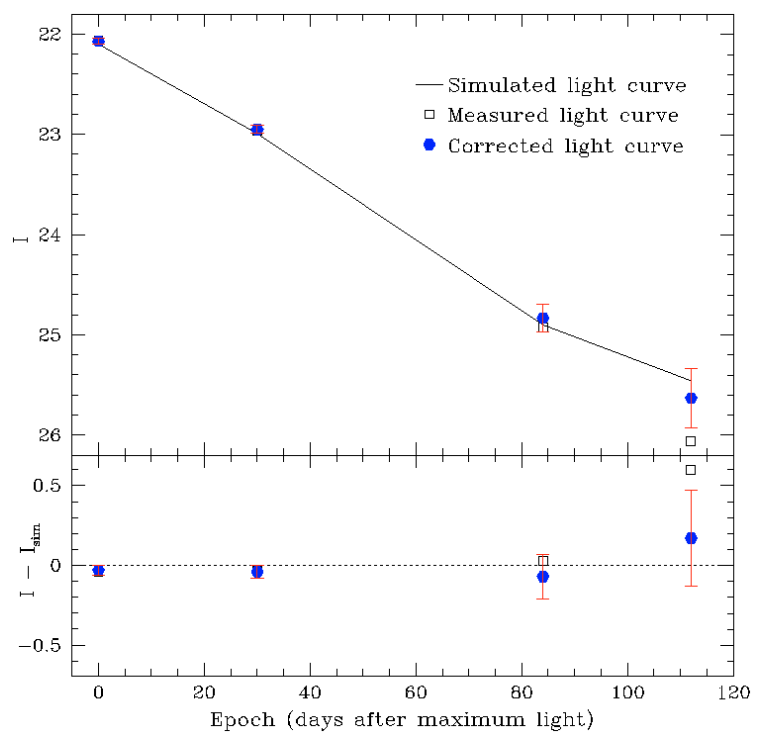

Fig. 10. "Observed" light curve of a simulated type Ia supernova at $z=$ 0.5 . Solid line is the model light curve in the VIMOS $I$ band from peak magnitude to $\sim 120$ days (in the observers frame). Open (black) squares show the measured SN magnitudes in the subtracted frames and solid (blue) hexagons show the corrected magnitudes (see text for details). The error bars show the scatter measured by the detailed simulations reported in Sect. 5.1, and are shown as $\pm 1 \sigma$ error bars to the corrected magnitudes. For the brightest magnitudes the errors are smaller than or roughly the size of the corrected points and thus not visible in the plot.

are not always rejected in the stamp selection by ISIS. This is a problem for the automatic stamp selection method where the subtraction quality can go down considerably. With the other methods the stamp list can always be manually edited before the subtraction and the non-suitable stamps removed from the list.

\subsection{Estimating photometric errors for SN light curves}

Running the detection pipeline on simulated supernovae can provide useful estimates of possible systematic offsets as well as photometric errors. Figure 10 shows the light curve of a type Ia supernova at redshift 0.5 (light curve model constructed using the templates provided by Nugent ${ }^{1}$ ). The measurements were done in the subtracted images using PSF fitting photometry (as discussed in Sect. 2.4). Using the simulations reported in Sect. 5.1 we have corrected the light curve for the systematic flux offset and estimated the uncertainty for each epoch measurement. Since the pixel to pixel noise in the subtracted images is correlated the magnitude errors reported by daophot will not be reliable (they will underestimate the errors). The uncertainties are instead estimated as the scatter of the simulated magnitude bins (see Fig. 5, interpolating when necessary). The systematic offset and the scatter will be different for each epoch, thus simulations need to be carried out in each epoch. These realistically estimated errors are on the order of 2-5 times higher than the standard output from daophot.

The correction for the systematic flux offset is calculated as a mean quantity for a large number of $\mathrm{SNe}$. For the case shown in Fig. 10 the corrected magnitudes is a better match to the simulated light curve than the uncorrected ones, the mean residual for the uncorrected and corrected curve is 0.175 and 0.0775 , respectively. It is evident that the small flux offset is negligible for

\footnotetext{
${ }^{1}$ http://supernova.1bl.gov/ nugent/nugent_templates. html
}

the brighter points on the light curve and it is mainly the faintest point that is affected by the correction (also the one giving rise to most of the change in mean residual). It should also be noted that once the faintest data point has been corrected it is consistent (i.e. within $1 \sigma$ ) with the simulated light curve.

\section{Discussion}

\subsection{Overall pipeline performance}

We have studied the detection efficiency and photometric accuracy of our SN detection pipeline using real data from the SVISS. However, these results can likely be applied to any SN ground based imaging survey using ISIS subtracted images for detection and/or photometry. Previous tests of image subtraction software have been done mainly on artificial images (AL98; A00; Gössl \& Riffeser 2002; Israel et al. 2007) but also on stellar fields (AL98). To our knowledge the work presented in this paper is the first detailed study of subtraction quality in real supernova survey data. Detection efficiency studies in supernova survey data are regularly carried out to be able to find the supernova rates (e.g. Cappellaro et al. 2005; Barris et al. 2005; Miknaitis et al. 2007), but this testing is seldom reported in detail.

We have found a systematic flux offset affecting the photometry in subtracted images. In general, with host galaxies chosen at random in the field, the offset is not dependent on the SN brightness, the offset is constant for different simulated SN magnitude bins. The offset is only found for SNe placed inside host galaxies whereas in isolated positions no offset is found. The offsets change between the different epochs, in some cases being positive and in others negative. Pixel statistics for pixels inside apertures centred on the host galaxies also show that there is a shift of the median pixel value in the same direction as the flux difference plots indicate and as expected, a broadening of the distribution. We interpret this as a small flux through/plateau at the locations of galaxies, reaching as far out as to the $80 \%$ flux radius. Currently we do not have an explanation for this effect, it cannot be solved by changing the convolution kernel sizes or stamp sizes. Further testing is needed to pinpoint if the systematic offset is caused by properties of the dataset or if it is in fact an internal effect of the image subtraction method. It should be noted that this is a small offset, the systematic error on the magnitudes of $\mathrm{SNe}$ is smaller for brighter sources (for the brightest SNe considered in this paper, $m_{I}=22.0$, the offset is $\sim 0.005$ ) and in general significantly smaller than the photometric scatter for an individual source. However, in a survey of multiple objects, and particularly fainter objects, this systematic effect will likely affect the result unless dealt with.

A simple estimate of the photometric uncertainties using the pixel to pixel noise in the local background underestimates the errors when doing photometry on SNe in subtracted images. To get an accurate noise estimate we have instead computed the photometric scatter for each simulated magnitude bin. This scatter has also been used to construct signal to noise ratios, using this method the $S N R$ is not affected by the flux offset and provides a simple way of rejecting less trustworthy observations.

Miknaitis et al. (2007) use simulations to check whether image registration and subtraction affect their SN photometry. They find no systematic offsets to a $S N R$ of 5 , but this requires an accurate SN position obtained from multiple points on the light curve. They find the photometric scatter to be on the order of $20 \%$ larger than what their noise maps indicate (but the noise maps already include the effects of re-binning and convolution). This seems considerably better than the accuracy we find using 
our pipeline and simulations on the SVISS data. The method used by these authors is quite different from the one used in this work, therefore it is hard to compare the exact figures. We estimate the $S N R$ s differently by using the scatter found from simulations as our noise. It should also be noted that the SN positions in the images used by Miknaitis et al. (2007) are chosen at random (private correspondence Miknaitis), thus their results will not be directly comparable to our inside host galaxies simulations. The main difference is that we find that subtraction residuals from the host galaxies can have a noticeable effect on the photometric accuracy of the survey. This effect does not seem to be present in the work of Miknaitis et al. (2007).

The presence of spurious detections is a problem for all supernova surveys, and in particular for surveys with spectroscopic follow-up (see the discussion in Bailey et al. 2007). For multiband, multi-epoch imaging surveys (like the SVISS) the spurious detections is less of a problem, but it is still desired to keep the number down. The bright residuals found in less successful image subtractions can easily be mistaken for real varying sources when using automatic source detection. The number of spurious detections in our tests is large, partly because we use a low detection threshold, but in general we find that a single epoch, single filter run results in a spurious detection rate of around $80 \%$ (i.e. 1 out of 5 are real sources) when the detection threshold is set to find objects brighter than the $5 \sigma$ limiting magnitude. For the SVISS dataset used in this paper the total number of detected sources for a given epoch is on the order of hundreds, making the number of spurious detections quite large. We find that the use of a controlled and bright stamp list makes the number of spurious detections go down by about $10 \%$. It should be noted that residuals at the locations of bright galaxies will be problematic even in a multi-band survey since the residuals often appear at the same positions in all filters.

\subsection{Host galaxy brightness and supernova position}

We find that the typical brightness of the host galaxies does affect the detection efficiencies and photometric accuracy. This is of course as expected and also confirmed by other SN surveys (e.g. Neill et al. 2006). However, both detection efficiency and photometric accuracy becomes gradually worse when considering host galaxies of increasing brightness. The effect is mainly noticeable for the very brightest host galaxies (i.e. largest contrast between the SN and the host). The fact that the photometry is affected by host brightness can in principle cause the systematic offset found above to vary with the SN brightness, since the SN and host brightness are correlated through the host galaxy distance. In projects where the supernova rate is studied, the effects of host brightness most likely have an impact on the detection efficiencies at low redshifts, where the host galaxies can be very bright, as well as in bright galaxies at high redshifts where the supernovae will be very faint. Whether this effect will be noticeable depends on the characteristics of the galaxy population, and simulations of the type reported in this work can successfully quantify the effect for galaxies of different brightness. This information can then be incorporated into the overall detection efficiencies discussed above. The SNe detections in the SVISS data are likely to be found in faint galaxies and thus in the general case not affected strongly by the host background flux.

The effect of the SN galactocentric distance in the host galaxy is difficult to study in the SVISS data set, since most of the galaxies have small angular sizes, and in many cases are not even resolved. Our results indicate that the flux offset is slightly worse in the inner parts ( $40 \%$ in flux units) of the galaxies than in the outer (40-80\%) regions. Therefore, we cannot draw any conclusions on how the detection efficiencies are affected by the galactocentric distances. However, if the detection efficiency is dependent on radial distance from the host centre, the radial distribution function of $\mathrm{SNe}$ needs to be known to find a proper correction factor for the total efficiency.

\subsection{Image subtraction method}

Of the different tested parameters we find that the stamp selection method affects the subtraction quality and detection success the most. The automatic stamp selection in ISIS mostly works fine, but the lack of control in selecting stamps can be problematic when dealing with real SN survey data, due to the presence of saturated objects and the rejection routine sometimes failing for varying objects. Using a set of sub-optimal stamps will result in a subtracted frame with worse subtraction quality. We note that the optimal selection for stamps is based on $S N R$, selecting preferentially point-like sources as stamps, i.e. in general fainter sources than in the $S N R$ based selection, degrades the subtraction quality. Varying the widths of the Gaussian basis functions of the kernel within a reasonable range does not significantly alter the detection results, however it should be noted that if all of the functions are too wide, depending on the data in question the image subtraction will fail. We also note that for surveys with small seeing differences, like the SVISS, the default kernel widths used in ISIS might be to wide. Using widths more in line with the theoretically optimal convolution kernel width can marginally improve the subtraction quality.

\subsection{Implications for $S N$ surveys}

The detection efficiency of supernovae in a multi-epoch survey can be studied by Monte Carlo simulations of SN light curves as observed by the telescope used for the survey (see e.g. Dahlen et al. 2004). The light curves, together with the individual detection efficiencies for each epoch can then be used to find an overall detection efficiency for supernovae of a certain type and at a given redshift. The flux offset effect found in this work will affect the detection limits as well as the number of spurious detections. These can be handled in different ways, but their presence will always be a problem and minimising the number of spurious detections is frequently important. A positive flux offset will result in an artificially boosted detection efficiency and an increase in the number of spurious detections. A negative offset will instead cause the detection efficiency to go down, making the survey to lose depth. This effect can possibly be countered by controlling the detection threshold used by SE using a weight map, in principle redefining the threshold for positions considered to be inside galaxies.

It is very unlikely that systematic flux offsets of the kind found in this work affect the results found by the large SN Ia projects. In these projects (e.g. Barris \& Tonry 2006; Astier et al. 2006; Miknaitis et al. 2007), the photometric accuracy is studied extensively to make sure that the systematic errors in the cosmological parameter determination is minimised. As shown above the detection efficiency will be influenced by the flux offset. Projects studying the supernova rate and doing detections in images subtracted using methods similar to ours might suffer from this effect and detailed simulations should be run to make sure that no offset is present (or correct for it otherwise). The results from some recent surveys (Dahlen et al. 2004; Cappellaro et al. 2005; Barris \& Tonry 2006) are based on supernovae with observed spectroscopy. The limiting magnitudes for 
these surveys are thus set by the magnitude limit of the spectroscopic instrument used, which is considerably brighter than the corresponding imaging limit, thus the offset problem described here is likely not an issue. However, with present and upcoming surveys based on imaging alone and pushing the detections to the fainter limits this effect needs to be understood and studied thoroughly.

Based on the findings reported in this paper we can make some recommendations for supernova detection and photometry using image subtraction methods. Testing should be done on at least part of the data to determine optimal parameters for the image subtraction. The stamp selection should be controlled in some way, a good option is to create a master stamp list from the reference image and use that in all subtractions but making sure that none of the stamps contain SNe. If the automatic stamp selection in ISIS is used, care must be taken that the selected stamps are not variable or contain saturated pixels. To make sure that a possible flux offset will not affect the results and to estimate photometric errors, simulations should be done. If photometry is done in the subtracted frames, the measurements for an individual SN should be carefully done. This can be done by simulating SNe of similar magnitude in nearby galaxies of the same brightness and size as the host galaxy and by measuring the possible offset. The photometric errors for each point on the light curve should be estimated by using the magnitude scatter found from simulations. If no offset correction for the detection efficiencies is applied we recommend that the detection efficiencies should be capped by the efficiency for isolated $\mathrm{SNe}$ (in the presence of a positive flux offset), the true efficiency can never be better inside than outside galaxies.

\section{Summary}

We have described the SN detection and photometry pipeline used for the SVISS. We have also presented detailed testing of supernova detections and photometry using image subtraction and automatic detection. Our main results and conclusion are:

- Image subtraction using ISIS 2.2 works well for the SVISS, the subtraction quality as measured by noise levels in the location of galaxies is in general good. Simulations show that our pipeline is successful in detecting $\mathrm{SNe}$ as well as in doing photometry on them.

- The detection efficiency and photometric accuracy for supernovae in a single epoch is affected by how the stamp selection for ISIS is done. We have also found that the host galaxy brightness does affect these quantities, likely through the presence of powerful subtraction residuals at the location of bright galaxies.

- Image subtraction using ISIS benefits from using a controlled stamp list. We have found that the best subtraction quality is achieved when the subtraction stamps used have high signal-to-noise ratios, ideally the brightest non-varying sources in the field should be used as stamps. The number of stamps depends on the size and quality of the image, and for our data set $\sim 60$ stamps per $(1 \mathrm{k} \times 1 \mathrm{k})$ pixels has proven to be sufficient.

- We have discovered a systematic flux offset in subtracted images. This offset only appears at the locations of galaxies and is quite small. Photometry of simulated $\mathrm{SNe}$ in isolated positions show no offset. For individual sources the offset is smaller than the estimated photometric scatter. This effect can cause systematic errors in the photometry if not corrected for. Detection limits of SNe will also be affected by non-zero offset, causing problems with increased numbers of spurious detections for positive offsets and a decrease in survey depth for a negative offset. It should be noted that, based on the tests run so far, we cannot say for sure whether the offset is something unique to the SVISS data set or if it is due to the image subtraction method.

- Simulations of supernovae both in and outside galaxies are needed to obtain proper estimates of the photometric errors. The simulations can also be used to correct for possible systematic offsets.

- Using automatic source detection (in this case SE) we find that the detection efficiencies can be pushed to the limits of the data. The number of spurious detections in a single epoch subtraction is very large, on the order of $80 \%$, but decreases as a function of subtraction quality. To reject spurious detections other methods must be used (e.g. multiple filters, light curve fitting).

Understanding possible systematic effects of the subtraction and detection process is very important when interpreting the results from the SVISS, but will also be imperative for the upcoming supernova projects based on large imaging surveys, e.g. PanSTARRS $^{2}$, LSST $^{3}$ and SNAP (Aldering 2005).

Acknowledgements. We would like to thank J. Sollerman, M. Hayes, Tomas Dahlen for helpful conversations and comments. We thank P. Price for useful discussions on the use of the image subtraction code. We would also like to thank the anonymous referee for many helpful and insightful comments and suggestions. Part of this work was based on observations done in service mode at the VLT/VIMOS instrument at Paranal Observatories.

We are grateful for financial support from the Swedish Research Council. S.M. acknowledges financial support from the Academy of Finland (project:8120503).

\section{References}

Alard, C. 2000, A\&AS, 144, 363 (A00)

Alard, C. 2001, MNRAS, 320, 341

Alard, C., \& Lupton, R. H. 1998, ApJ, 503, 325

Aldering, G. 2005, New Astron. Rev., 49, 346

Astier, P., Guy, J., Regnault, N., et al. 2006, A\&A, 447, 31

Bailey, S., Aragon, C., Romano, R., et al. 2007, ArXiv e-prints, 705

Barris, B. J., \& Tonry, J. L. 2006, ApJ, 637, 427

Barris, B. J., Tonry, J. L., Novicki, M. C., \& Wood-Vasey, W. M. 2005, AJ, 130, 2272

Bertin, E., \& Arnouts, S. 1996, A\&AS, 117, 393

Botticella, M. T., Riello, M., Cappellaro, E., et al. 2008, A\&A, 479, 49 Cappellaro, E., Riello, M., Altavilla, G., et al. 2005, A\&A, 430, 83

Corwin, T. M., Sumerel, A. N., Pritzl, B. J., et al. 2006, AJ, 132, 1014

Dahlén, T., \& Fransson, C. 1999, A\&A, 350, 349

Dahlen, T., Strolger, L.-G., Riess, A. G., et al. 2004, ApJ, 613, 189

Giacconi, R., Rosati, P., Tozzi, P., et al. 2001, ApJ, 551, 624

Gössl, C. A., \& Riffeser, A. 2002, A\&A, 381, 1095

Holman, M. J., Winn, J. N., Fuentes, C. I., et al. 2007, ApJ, 655, 1103

Israel, H., Hessman, F. V., \& Schuh, S. 2007, Astron. Nachr., 328, 16

La Franca, F., Gruppioni, C., Matute, I., et al. 2004, AJ, 127, 3075

LeFevre, O., Saisse, M., Mancini, D., et al. 2003, in Instrument Design and Performance for Optical/Infrared Ground-based Telescopes, Presented at the Society of Photo-Optical Instrumentation Engineers (SPIE) Conf., ed. M. Iye, \& A. F. M. Moorwood, Proc. SPIE, 4841, 1670

Maiolino, R., Vanzi, L., Mannucci, F., et al. 2002, A\&A, 389, 84 Mattila, S., \& Meikle, W. P. S. 2001, MNRAS, 324, 325

Mattila, S., Meikle, W. P. S., \& Greimel, R. 2004, New Astron. Rev., 48, 595

Mattila, S., Väisänen, P., Farrah, D., et al. 2007, ApJ, 659, L9

Meikle, W. P. S., Mattila, S., Gerardy, C. L., et al. 2006, ApJ, 649, 332

Miknaitis, G., Pignata, G., Rest, A., et al. 2007, ArXiv Astrophysics e-prints

Neill, J. D., Sullivan, M., Balam, D., et al. 2006, AJ, 132, 1126

Poznanski, D., Maoz, D., Yasuda, N., et al. 2007, MNRAS, 382, 1169

Sollerman, J., Holland, S. T., Challis, P., et al. 2002, A\&A, 386, 944

Sumi, T., Woźniak, P. R., Udalski, A., et al. 2006, ApJ, 636, 240

\footnotetext{
2 http://pan-starrs.ifa.hawaii.edu

3 http://www. lsst.org/lsst_home.shtml
} 